\title{
Grumiplucite from the Rudňany deposit, Slovakia: a second world- -occurrence and new data
}

\author{
Martin ŠTEVKO ${ }^{*}$, Jiří SEJKORA², Dušan PETEREC ${ }^{3}$ \\ ${ }^{1}$ Comenius University, Faculty of Natural Sciences, Department of Mineralogy and Petrology, Ilkovičova 6, 84215 Bratislava, Slovakia; \\ stevko@fns.uniba.sk \\ 2 Department of Mineralogy and Petrology, National Museum, Cirkusová 1740, 19300 Prague 9, Czech Republic \\ ${ }^{3}$ Rovníková 8, 04012 Košice, Slovakia \\ * Corresponding author
}

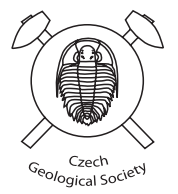

Grumiplucite, ideally $\mathrm{HgBi}_{2} \mathrm{~S}_{4}$ was identified at the Droždiak vein, Rudñany deposit (Spišsko-gemerské Rudohorie Mts., Slovakia). This rare $\mathrm{Hg}$-sulfosalt forms metallic lead-grey to steel-grey, prismatic to acicular crystals up to $1 \mathrm{~cm}$ long, often grouped into irregular aggregates. It occurs in cavities of siderite with abundant cinnabar, Hg-rich tetrahedrite and chalcopyrite aggregates. Minor quartz or barite crystals and microscopic aggregates of Sb-rich bismuthinite to Birich antimonite were also observed. On the basis of chemical analyses, two types of grumiplucite were distinguished at the Rudňany deposit. The first is close to an ideal composition and has empirical formula $\mathrm{Hg}_{0.99} \mathrm{Bi}_{1.94} \mathrm{~S}_{4.08}$ (based on 7 $a p f u)$. The second is characterized by regularly elevated contents of $\mathrm{Sb}$ ranging from 0.02 to 0.77 apfu. Grumiplucite is monoclinic, space group $C 2 / m$, with unit-cell parameters refined from X-ray powder data: $a=14.172(2), b=4.0525(7)$, $c=13.975(1) \AA, \beta=118.257(8)^{\circ}$ and $V=707.0(2) \AA^{3}$ (Sb-free) and $a=14.183(1), b=4.0538(5), c=13.980(1) \AA, \beta=$ $118.239(1)^{\circ}$ and $V=708.1(2) \AA^{3}$ (Sb-rich). Raman spectra for grumiplucite crystals with variable Sb contents are mutually well comparable. Therefore it seems that different $\mathrm{Sb}$ occupation does not affect the energy and intensity of Raman bands and they significantly differ from spectra of particularly structure-related mineral livingstonite, $\mathrm{HgSb}_{4} \mathrm{~S}_{8}$. The dominant feature in the Raman spectra of grumiplucite is a series of spectral bands that corresponds to the stretching and bending vibrations of $\mathrm{BiS}_{5}$ polyhedra and $\mathrm{Hg}-\mathrm{S}$ bonds.

Keywords: grumiplucite, sulfosalts, X-ray powder data, chemical composition, Raman spectroscopy, Rudñany deposit Received: 23 July, 2015; accepted: 1 December, 2015; handling editor: F. Laufek

\section{Introduction}

Grumiplucite, $\mathrm{HgBi}_{2} \mathrm{~S}_{4}$, is a very rare sulfosalt, a member of the pavonite homologous series, which was described as a new species from Levigliani mercury mine, Apuan Alps, Tuscany, Italy. It occurs there as prismatic, greyblack metallic crystals up to $1 \mathrm{~mm}$ in size, associated with cinnabar, native mercury, Hg-rich sphalerite and pyrite in cavities of the quartz-carbonate veins (Orlandi et al. 1998). Its crystal structure (Fig. 1) has an order-disorder (OD) character and consists of (001) layers, which are built up by $\mathrm{Bi}_{2} \mathrm{~S}_{4}$ rods of edge-sharing square-pyramidal $\left[\mathrm{BiS}_{5}\right]$ polyhedra. These $\mathrm{Bi}_{2} \mathrm{~S}_{4}$ rods are running along the $b$ axis and are interconnected through $\mathrm{Hg}^{2+}$ ions in the [100] direction. Adjacent layers are connected to each other through weak $\mathrm{Bi}-\mathrm{S}$ bonds. Two possible maximum degree of order (MDO) polytypes were distinguished; the first $\left(\mathrm{MDO}_{1}\right)$ orthorhombic with the space group $\mathrm{Ccm} 2_{1}$ and the second $\left(\mathrm{MDO}_{2}\right)$, corresponding to the natural grumiplucite, monoclinic with the space group $C 2 / m$ (Merlino et al. 2013).

Up to now grumiplucite has been described only from the type locality (Orlandi et al. 1998; Merlino et al.
2013). This paper is focused on detailed mineralogical characterization of grumiplucite and associated minerals from the Rudňany deposit, Spišsko-gemerské Rudohorie Mts., Slovakia, which is a second world occurrence of this $\mathrm{Hg}$-sulfosalt. We present here new wavelength-dispersive electron probe microanalyses, X-ray powder diffraction and Raman data for this very rare mineral phase.

\section{Geological setting}

The Rudňany deposit (or Rudňany ore field) is located c. $11 \mathrm{~km} \mathrm{SE}$ of Spišská Nová Ves town in the northern part of Gemeric Superunit, Spišsko-gemerské Rudohorie Mts., Slovakia (Fig. 2); GPS coordinates of the Západ shaft are: $48^{\circ} 52^{\prime} 47.79^{\prime \prime} \mathrm{N}$ and $20^{\circ} 39^{\prime} 29.98^{\prime \prime} \mathrm{E}$. It was one of the most important mining centers as well as the largest siderite-type ( $\mathrm{Fe}-\mathrm{Ba}-\mathrm{Cu}-\mathrm{Hg})$ deposits in Slovakia, mined already in prehistoric times. Most of the mining activity ceased there in 1993; only minor mining of barite still continues at the Poráč shaft area to the present day.

Hydrothermal siderite-quartz \pm barite veins, sometimes with abundant sulfidic mineralization, strike W-E 


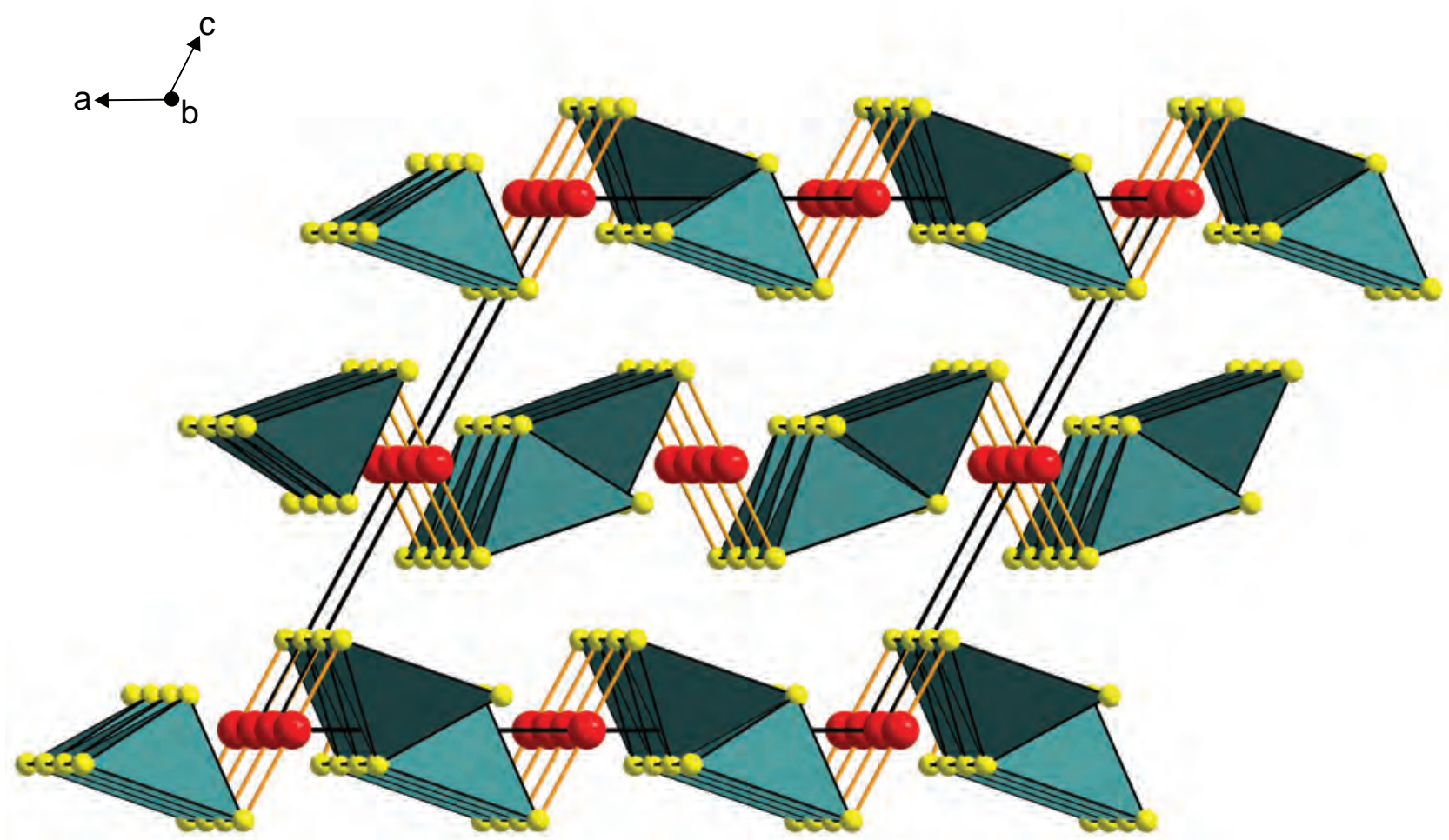

Fig. 1 The crystal structure of synthetic analogue of grumiplucite (Mumme and Watts 1980) viewed along the $b$ axis. Edge-sharing square-pyramidal $\left[\mathrm{BiS}_{5}\right]$ polyhedra are green, $\mathrm{S}$ atoms yellow and $\mathrm{Hg}$ atoms red. Unit-cell edges are highlighted.

and are in the central parts hosted by Carboniferous metasediments (conglomerates, sandstones and black shales) of the Dobšiná Group and in the lower parts by Early Paleozoic rocks (phyllites, metabasalts and amphibolites) of the Rakovec Group. Only apical parts of veins occur in Permian conglomerates and sandstones that belong to the Krompachy Group (Cambel et al. 1985; Vozárová and Vozár 1988; Grecula et al. 1995). From south to north, the Zapálenica, Droždiak, Hrubá, Štefan, Zlatník, Severná, Matej, Jakub, Ján, Lendava and Miloj veins were mined or explored at the Rudňany deposit. With a length of $7 \mathrm{~km}$, vertical extent more than $900 \mathrm{~m}$ and thickness from 7 to $40 \mathrm{~m}$, the Droždiak vein is the largest siderite vein in the Carpathian region (Cambel et al. 1985; Grecula et al. 1995).

Siderite, ankerite, barite and quartz are the most common gangue minerals at the Rudňany deposit. Ore minerals are irregularly distributed and are represented mainly by chalcopyrite, pyrite, tetrahedrite, cinnabar, arsenopyrite, Ni-Co sulfoarsenides and arsenides and hematite (Bernard 1955, 1961; Hurný and Krištín 1978; Cambel et al. 1985). In total, more than 100 minerals were described from the Rudňany deposit up to date (Ďud'a and Ozdín 2012).

The siderite veins at the Rudnany deposit are vertically zoned; barite prevails in the upper parts, siderite in the central and quartz-sulfidic mineralization in the lower parts (Bernard 1961; Rojkovič 1977; Cambel et al. 1985). According to Rojkovič (1977) and Cambel et al. (1985), the vein infill was formed at two mineralization stages: a siderite stage, with fuchsite and siderite-barite phases, and a quartz-sulfide stage with quartz-tourmaline, sulfide and cinnabar phases.

Two principal genetic models were adopted for the origin of the siderite veins in the Gemeric Superunit. Žák et al. (1991) and Radvanec et al. (2004) assumed that the veins were formed during multistage hydrothermal circulation of metamorphic fluids and their possible mixing with meteoric waters leaching Permian evaporites. In this model, Variscan mineralization phase was considered dominant, while the Alpine mineralization phase was only minor. On the other hand, according to Hurai et al. $(1998,2002)$, siderite veins in the Gemeric Superunit originated from basinal brines expelled during compression, which was associated with a continental collision of the Alpine Orogeny.

\section{Analytical methods}

The samples studied in this work were collected at the mining stope No. 9203, the Droždiak vein, $19^{\text {th }}$ level of the Západ shaft at the Rudňany deposit in 1993. The Droždiak vein is strongly tectonically deformed in this 


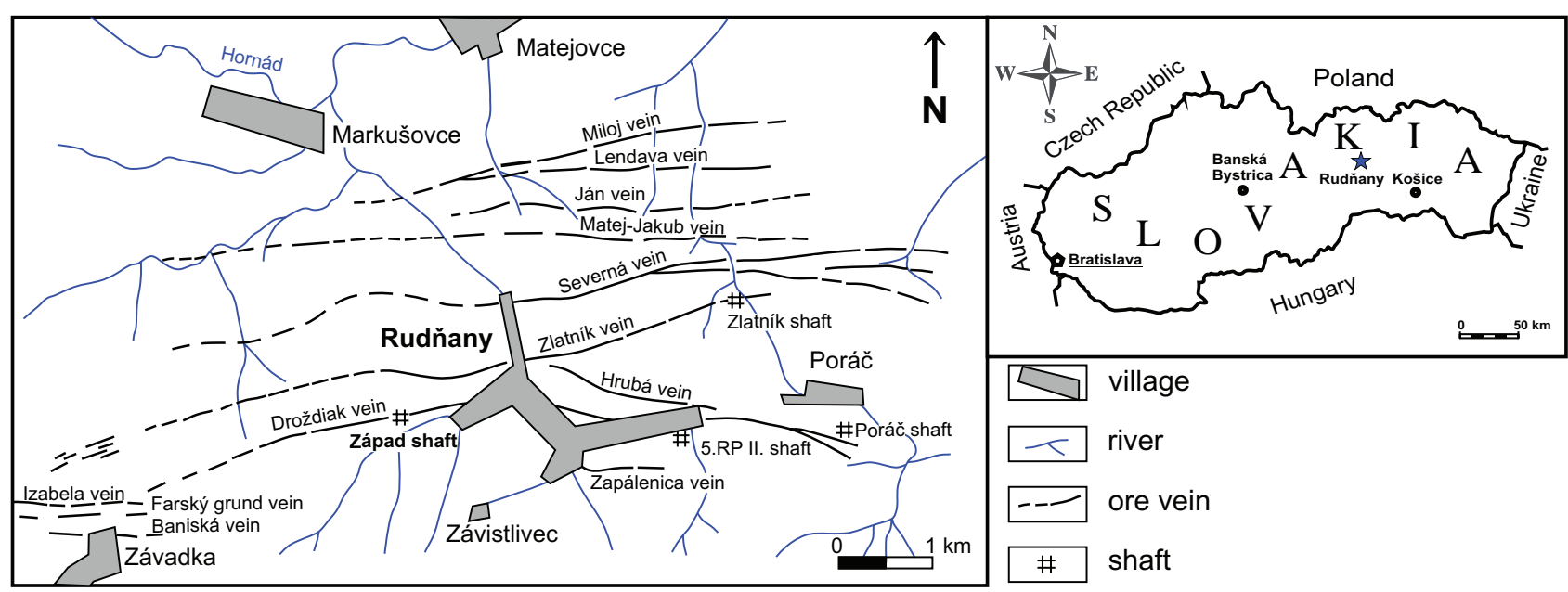

Fig. 2 Schematic sketch of the Rudňany deposit with distribution of ore veins (modified after Grecula et al. 1995).

area and segmented to several separate vein structures. Samples with grumiplucite originated from one of them, known as the Stredná vein, which was particularly rich in sulfides, especially chalcopyrite, tetrahedrite and cinnabar.

The specimens with grumiplucite were documented by Canon EOD 5D Mark II digital camera with macro lens employing multifocal processing. Depth of focus of the photos was controlled by stacking a number of co-axial pictures using the Helicon Focus software. Details of grumiplucite morphology were studied in low-vacuum mode of the Hitachi S-3700 N scanning electron microscope (National Museum, Prague). Polished sections were prepared for optical investigation and chemical analysis using standard diamond-polishing techniques. Optical properties in reflected light were observed with a Nikon Eclipse ME600 microscope (National Museum, Prague).

Quantitative chemical compositions of grumiplucite and associated minerals were determined with a Cameca SX100 electron-microprobe (National Museum, Prague), operated in the wavelength-dispersive mode at the following conditions: accelerating voltage of $25 \mathrm{kV}$, beam current of $10 \mathrm{nA}$ and electron-beam diameter of $5 \mu \mathrm{m}$. The standards used were (DL - detection limit): chalcopyrite $\left(\mathrm{SK}_{\alpha} \mathrm{DL} 0.03\right), \mathrm{Bi}_{2} \mathrm{Se}_{3}\left(\mathrm{Bi}_{\beta} \mathrm{DL} 0.16\right), \mathrm{PbS}\left(\mathrm{Pb} M_{\alpha}\right.$ DL 0.11), $\mathrm{Ag}\left(\mathrm{AgL} L_{\alpha} \mathrm{DL} 0.07\right)$, halite ( $\mathrm{Cl} K_{\alpha}$ DL 0.02), $\mathrm{Sb}_{2} \mathrm{~S}_{3}\left(\mathrm{Sb} L_{\alpha}\right.$ DL 0.05), CdTe (Cd $L_{\alpha}$ DL 0.11), HgTe $\left(\mathrm{Hg} M_{\alpha}\right.$ DL 0.12), pyrite ( $\left.\mathrm{Fe} K_{\alpha} \mathrm{DL} 0.05\right), \mathrm{Cu}\left(\mathrm{Cu} K_{\alpha}\right.$ DL 0.04), $\mathrm{ZnS}\left(\mathrm{Zn} K_{\alpha} \mathrm{DL}\right.$ 0.07), NiAs (As $\left.L_{\alpha} \mathrm{DL} 0.06\right)$ and $\mathrm{PbSe}$ $\left(\mathrm{Se} L_{\beta} \mathrm{DL} 0.08\right)$. Elements above that are not included in tables of analytical data were acquired but their contents were below detection limit. Measured data were corrected using PAP software (Pouchou and Pichoir 1985).

$\mathrm{X}$-ray powder diffraction patterns were obtained from hand-picked samples using a Bruker D8 Advance diffractometer equipped with solid-state 1D LynxEye detector using $\mathrm{Cu} K_{\alpha}$ radiation and operating at $40 \mathrm{kV}$ and $40 \mathrm{~mA}$ (National Museum, Prague). In order to minimize the background, the powdered samples were placed on the surface of a flat silicon wafer from acetone suspension. The powder patterns were collected in the Bragg-Brentano geometry in the range $4-75^{\circ} 2 \theta$, step $0.01^{\circ}$ and counting time of $30 \mathrm{~s}$ per step (total duration of experiments was $c .3$ days). The positions and intensities of diffractions were found and refined using the Pearson VII profile-shape function of the ZDS program package (Ondruš 1993) and the unit-cell parameters were refined by the least-squares program of Burnham (1962).

Raman spectra of grumiplucite from Rudňany and livingstonite from Huitzuco were collected in the range 30$3500 \mathrm{~cm}^{-1}$ using a DXR dispersive Raman Spectrometer (Thermo Scientific) mounted on confocal Olympus microscope (National Museum, Prague). The Raman signal was excited by a green $532 \mathrm{~nm}$ diode-pumped solid-state laser and detected by a CCD detector. The experimental parameters were: $50 \times$ objective, $1 \mathrm{~s}$ exposure time, 1000 exposures, 900 lines/mm grating, $50 \mu \mathrm{m}$ slit spectrograph aperture and $0.5 \mathrm{~mW}$ laser power level. The instrument was set up by a software-controlled calibration procedure using multiple neon emission lines (wavelength calibration), multiple polystyrene Raman bands (laser frequency calibration) and standardized white-light sources (intensity calibration). Spectral manipulations were performed using the Omnic 9 software (Thermo Scientific).

\section{Results and discussion}

\subsection{Grumiplucite}

Grumiplucite has been identified in few samples only. It forms well developed metallic lead grey to steel grey, prismatic to acicular crystals (Fig. 3) with visible striations and often twinned along (001) plane (Fig. 4a) and 


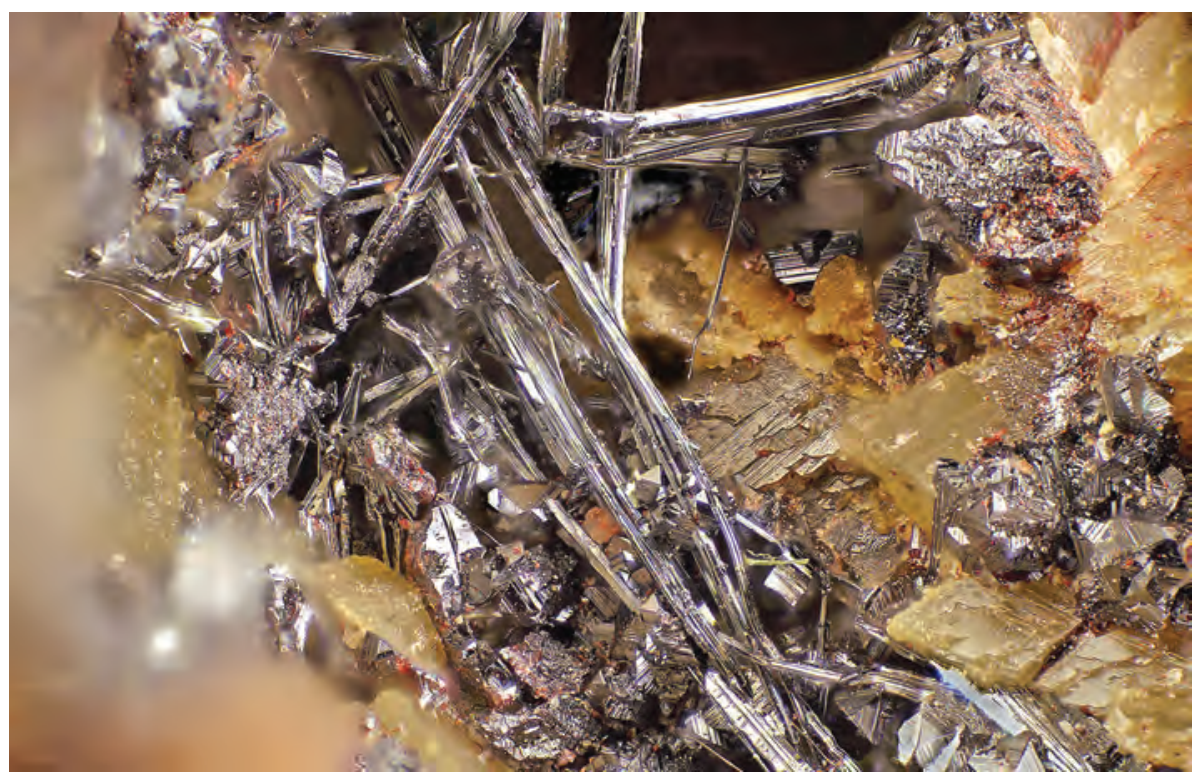

Fig. 3 Group of prismatic to acicular grumiplucite crystals associated with cinnabar and siderite crystals; width of image $4 \mathrm{~mm}$, photo P. Škácha. with perfect $\{001\}$ cleavage. Individual crystals reach mostly 2-4 $\mathrm{mm}$ in length; rarely crystals up to $1 \mathrm{~cm}$ were observed. They are often bent and grouped to irregular aggregates in cavities of coarse-grained siderite
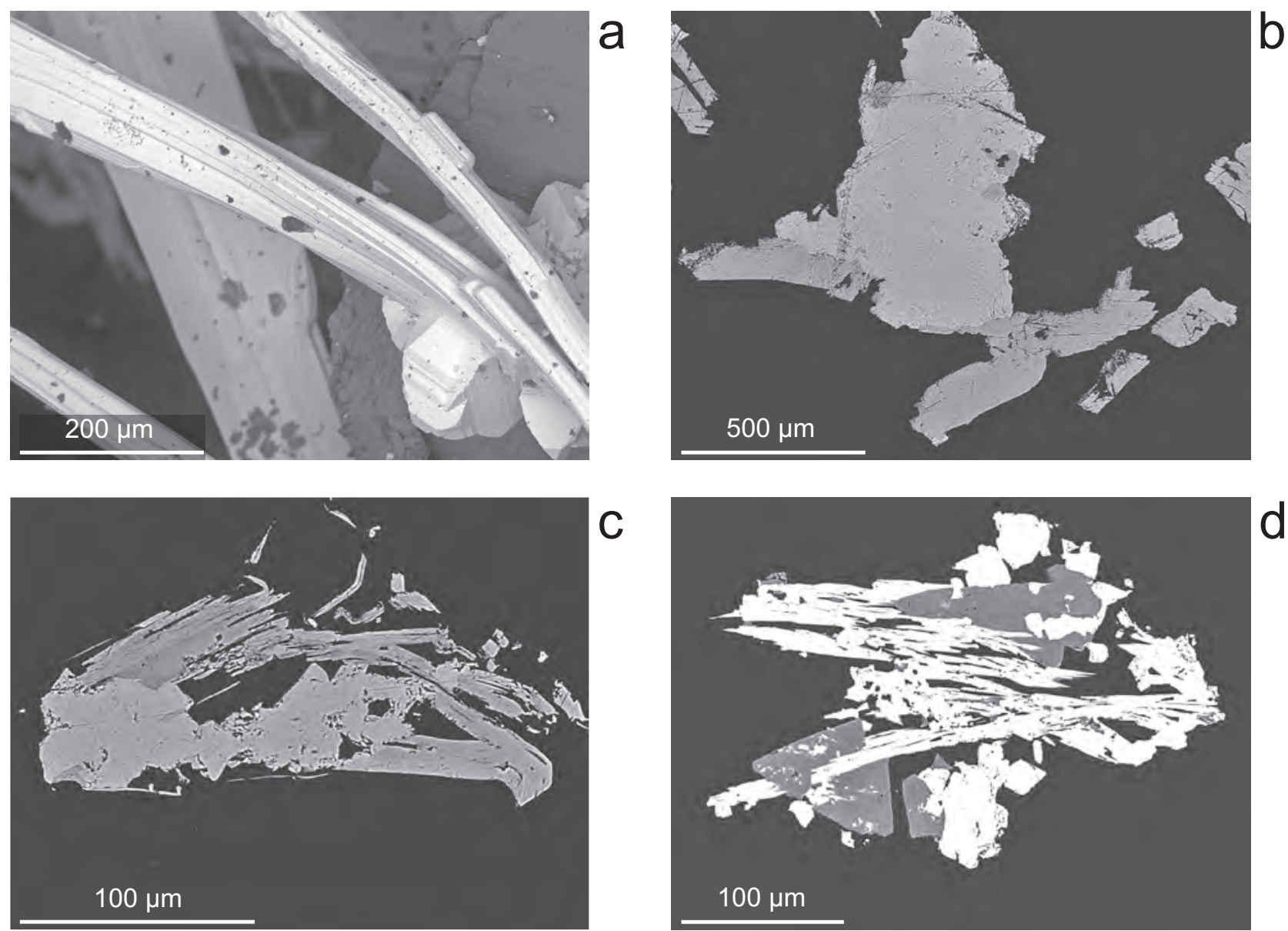

Fig. 4a - Detail on bend crystals of grumiplucite with well visible striations. b - Homogenous grumiplucite crystals (dark grey) with cinnabar (grey); BSE image. c - Grumiplucite crystals with variable Sb contents (dark grey) with cinnabar (grey); BSE image. d - Grumiplucite crystals (white) with later crystals of Hg-rich tetrahedrite (dark grey); BSE image. 
with abundant cinnabar, tetrahedrite and chalcopyrite and minor arsenopyrite grains. Grumiplucite is one of the youngest ore minerals at the Rudňany deposit, closely associated with later cinnabar crystals (Figs 3-4c) or crystals of $\mathrm{Hg}$-rich tetrahedrite (Fig. 4d). Minor quartz and barite crystals as well as microscopic aggregates of minerals of bismuthinite-stibnite solid solution (Fig. 5) were rarely also identified.

On the basis of quantitative chemical analyses, two types of grumiplucite were distinguished at the Rudňany deposit. The first and dominant type (Fig. 4b) has a homogenous composition (Tab. 1) close to the theoretical formula $\mathrm{HgBi}_{2} \mathrm{~S}_{4}$ or resembling the data published by Orlandi et al. (1998) for the material from the type locality. Only infrequent and subordinate amounts of $\mathrm{Cu}$ (up to $0.01 \mathrm{apfu}$ ) were detected in this type of grumiplucite. Its empirical formula (average of 34 point analyses) is

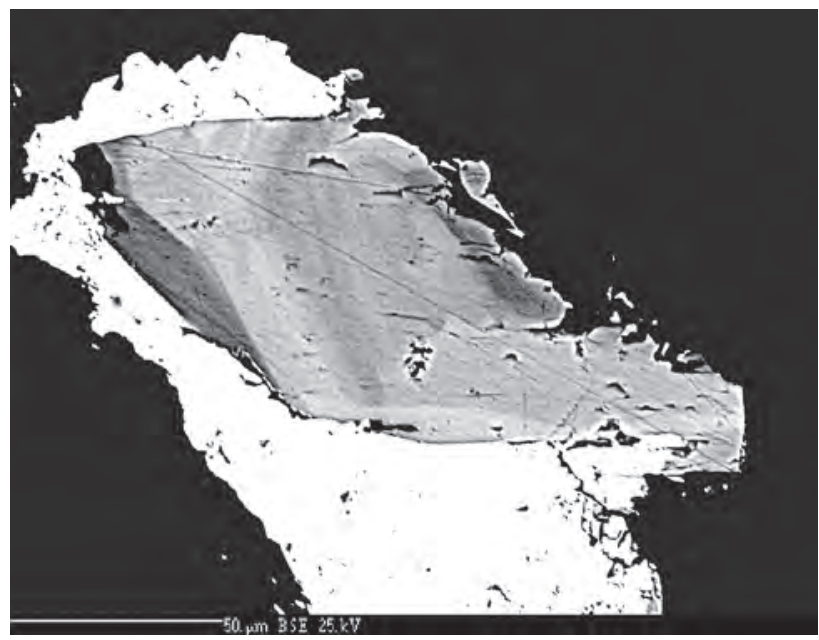

Fig. 5 Strongly zoned aggregate of Bi-rich stibnite with cinnabar (white); BSE image.

Tab. 1 Chemical composition of Sb-free grumiplucite from Rudňany (in wt. \% and apfu)

\begin{tabular}{|c|c|c|c|c|c|c|c|c|c|c|c|c|c|c|}
\hline & ideal $^{1}$ & type $^{2}$ & mean $^{3}$ & range $^{3}$ & 1 & 2 & 3 & 4 & 5 & 6 & 7 & 8 & 9 & 10 \\
\hline $\mathrm{Hg}$ & 26.86 & 25.40 & 26.70 & $26.19-30.05$ & 30.05 & 26.88 & 26.79 & 26.81 & 27.04 & 26.75 & 26.91 & 26.19 & 26.36 & 26.55 \\
\hline $\mathrm{Cu}$ & & & 0.01 & $0.00-0.08$ & 0.00 & 0.00 & 0.00 & 0.00 & 0.00 & 0.00 & 0.08 & 0.00 & 0.00 & 0.00 \\
\hline $\mathrm{Bi}$ & 55.97 & 57.60 & 54.67 & $51.30-55.43$ & 51.30 & 54.85 & 54.69 & 54.88 & 54.65 & 54.43 & 54.69 & 54.83 & 55.43 & 55.04 \\
\hline $\mathrm{S}$ & 17.17 & 17.40 & 17.66 & $17.26-17.91$ & 17.26 & 17.46 & 17.39 & 17.54 & 17.57 & 17.54 & 17.68 & 17.79 & 17.79 & 17.91 \\
\hline total & 100.00 & 100.40 & 99.04 & & 98.61 & 99.19 & 98.88 & 99.23 & 99.26 & 98.71 & 99.36 & 98.81 & 99.58 & 99.50 \\
\hline $\mathrm{Hg}$ & 1.000 & 0.938 & 0.985 & & 1.123 & 0.997 & 0.997 & 0.992 & 0.999 & 0.992 & 0.990 & 0.965 & 0.967 & 0.971 \\
\hline $\mathrm{Bi}$ & 2.000 & 2.042 & 1.936 & & 1.841 & 1.952 & 1.954 & 1.949 & 1.939 & 1.938 & 1.932 & 1.938 & 1.951 & 1.932 \\
\hline $\mathrm{S}$ & 4.000 & 4.020 & 4.077 & & 4.036 & 4.051 & 4.049 & 4.059 & 4.062 & 4.070 & 4.069 & 4.097 & 4.082 & 4.097 \\
\hline
\end{tabular}

1ideal: ideal composition of $\mathrm{HgBi}_{2} \mathrm{~S}_{4}$

2type: grumiplucite from type occurrence Levigliani mine, Italy (Orlandi et al. 1998)

${ }^{3}$ mean and range for 34 spot analyses of Sb-free grumiplucite from Rudňany (this paper), 1-10 representative spot analyses

Empirical formulae were calculated on the basis of 7 apfu.

$\mathrm{Hg}_{0.99} \mathrm{Bi}_{1.94} \mathrm{~S}_{4.08}$ based on 7 apfu. The second and by far rarer type of grumiplucite is characterized by regularly elevated contents of $\mathrm{Sb}$ (from 0.02 to 0.77 apfu - Fig. 6); individual zones with variable contents of $\mathrm{Sb}$ are parallel to the elongation of crystals (Fig. 4c). It also contains minor amounts of $\mathrm{Cu}$ (up to $0.11 \mathrm{apfu}$ ), $\mathrm{Pb}$ (up to 0.02 $a p f u$ ), As (up to $0.02 a p f u$ ) and Se (up to $0.01 \mathrm{apfu}$ ). The coefficients of empirical formulae for 15 representative analyses (from the all 60 measured) of Sb-rich grumiplucite from Rudňany are given in Tab. 2.

Fig. $6 \mathrm{Bi}$ vs. $\mathrm{Sb}(a p f u)$ graph for two types of grumiplucite from Rudňany.

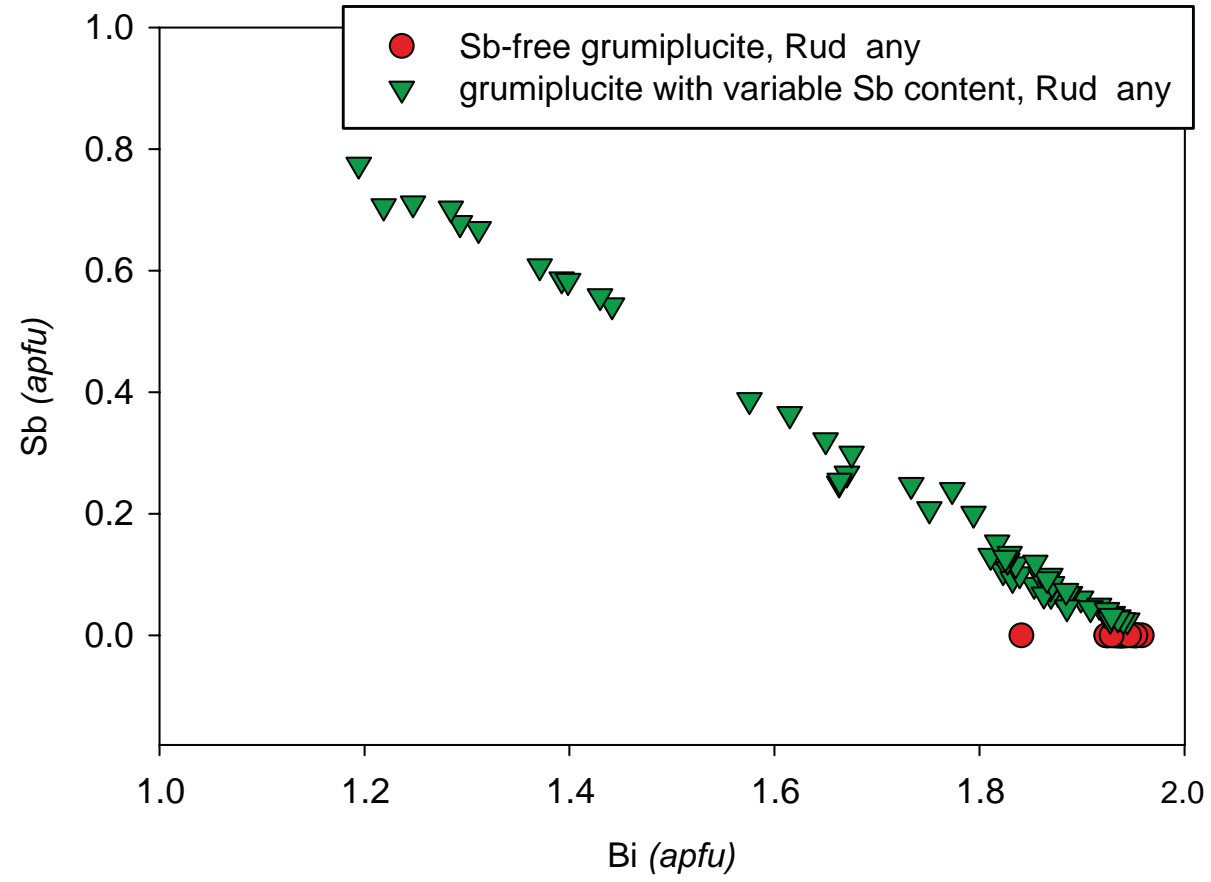


Tab. 2 Chemical composition of Sb-rich grumiplucite from Rudňany (in wt. \% and $a p f u$ )

\begin{tabular}{|c|c|c|c|c|c|c|c|c|c|c|c|c|c|c|c|}
\hline & 1 & 2 & 3 & 4 & 5 & 6 & 7 & 8 & 9 & 10 & 11 & 12 & 13 & 14 & 15 \\
\hline $\mathrm{Pb}$ & 0.53 & 0.34 & 0.51 & 0.24 & 0.34 & 0.00 & 0.19 & 0.20 & 0.37 & 0.17 & 0.00 & 0.00 & 0.13 & 0.00 & 0.00 \\
\hline $\mathrm{Hg}$ & 27.71 & 27.79 & 27.98 & 27.42 & 27.50 & 27.71 & 28.12 & 27.42 & 27.41 & 27.23 & 29.57 & 28.79 & 29.45 & 28.96 & 30.41 \\
\hline $\mathrm{Cu}$ & 0.00 & 0.07 & 0.11 & 0.06 & 0.06 & 0.00 & 12 & 0.08 & 0.05 & 0.18 & 0 . & 0.06 & 0.06 & 1.05 & 0.18 \\
\hline $\mathrm{Sb}$ & 0.42 & 0.45 & 0.53 & 1.20 & 1.76 & 1.98 & 2 & 2.22 & 2.52 & 3.95 & 4.25 & 6.65 & 11.69 & 12.90 & 13.81 \\
\hline $\mathrm{Bi}$ & 54.28 & 54.40 & 54.73 & 52.80 & 52.22 & 52.57 & 09 & 51.86 & .43 & 50.30 & 48.53 & 46.40 & 39.37 & 8.23 & 36.56 \\
\hline As & 00 & 00 & 00 & 00 & 00 & 00 & 0 & 0.00 & 00 & 0.00 & 0.00 & 0.00 & 0.00 & 0.25 & 0.00 \\
\hline Se & 0.00 & 0.00 & 0.00 & 0.00 & 0.00 & 0.09 & 0.10 & 0.00 & 0.00 & 0.10 & 0.00 & 0.12 & 0.00 & 0.00 & 0.00 \\
\hline $\mathrm{S}$ & 7.28 & 17.18 & 17.31 & 17.23 & 17.81 & 17.39 & 17.46 & 17.46 & 17.38 & 17.20 & 17.99 & 18.05 & 8.37 & 19.16 & 18.68 \\
\hline total & 100.22 & 0.24 & 101.17 & 94 & 99.70 & 99.75 & 100.20 & 99.23 & 99.16 & 99.13 & 100.47 & 100.06 & 99.06 & 100.56 & 99.65 \\
\hline $\mathrm{Pb}$ & 0.019 & 0.012 & 018 & 909 & 012 & 000 & 07 & 0007 & 12 & 0.006 & 0.000 & .000 & 0.004 & 0.000 & 0.000 \\
\hline $\mathrm{Hg}$ & 1.026 & 1.030 & 1.028 & 1.020 & 1.000 & 1.018 & 027 & 1.007 & 1.009 & 1.000 & 1.056 & 1.019 & 1.02 & 0.962 & 1.035 \\
\hline $\mathrm{Cu}$ & 0.000 & .008 & 0.013 & 0.007 & 0.006 & 0.000 & 014 & 0.009 & 0.005 & 0.021 & 0.014 & 0.006 & 0.006 & 0.110 & 0.019 \\
\hline $\mathrm{Hg}+\mathrm{Pb}+\mathrm{Cu}$ & 1.045 & 1.051 & 1.059 & 1.035 & 1.019 & 1.018 & 1.048 & 1.023 & 1.028 & 1.027 & 1.070 & 1.026 & 1.032 & 1.072 & 1.054 \\
\hline $\mathrm{Sb}$ & 0.026 & 0.028 & 0.032 & 0.073 & 0.106 & 0.120 & 0.128 & 0.134 & 0.153 & 0.239 & 0.250 & 0.388 & 0.669 & 0.706 & 0.774 \\
\hline $\mathrm{Bi}$ & 1.928 & 1.936 & 1.930 & 1.884 & 1.823 & 1.854 & 1.826 & 1.829 & 1.817 & 1.773 & 1.663 & 1.577 & 1.311 & 1.219 & 1.194 \\
\hline $\mathrm{Bi}+\mathrm{Sb}+\mathrm{As}$ & 1.954 & 1.964 & 1.962 & 1.958 & 1.929 & 1.974 & 1.954 & 1.963 & 1.970 & 2.013 & 1.913 & 1.965 & 1.980 & 1.947 & 1.969 \\
\hline Se & 0.000 & 0.000 & 0.000 & 0.000 & 0.000 & 0.008 & 0.009 & 0.000 & 0.000 & 0.009 & 0.000 & 0.011 & 0.000 & 0.000 & 0.000 \\
\hline $\mathrm{S}$ & 4.001 & 3.985 & 3.979 & 4.007 & 4.053 & 3.999 & 3.989 & 4.013 & 4.002 & 3.952 & 4.017 & 3.999 & 3.988 & 3.981 & 3.977 \\
\hline $\mathrm{S}+\mathrm{Se}$ & 4.001 & 3.985 & 3.979 & 4.007 & 4.053 & 4.007 & 3.998 & 4.013 & 4.002 & 3.961 & 4.017 & 4.010 & 3.988 & 3.981 & 3.977 \\
\hline
\end{tabular}

1-15: representative analyses of Sb-rich grumiplucite from Rudňany.

Empirical formulae were calculated on the basis of $7 \mathrm{apfu}$.

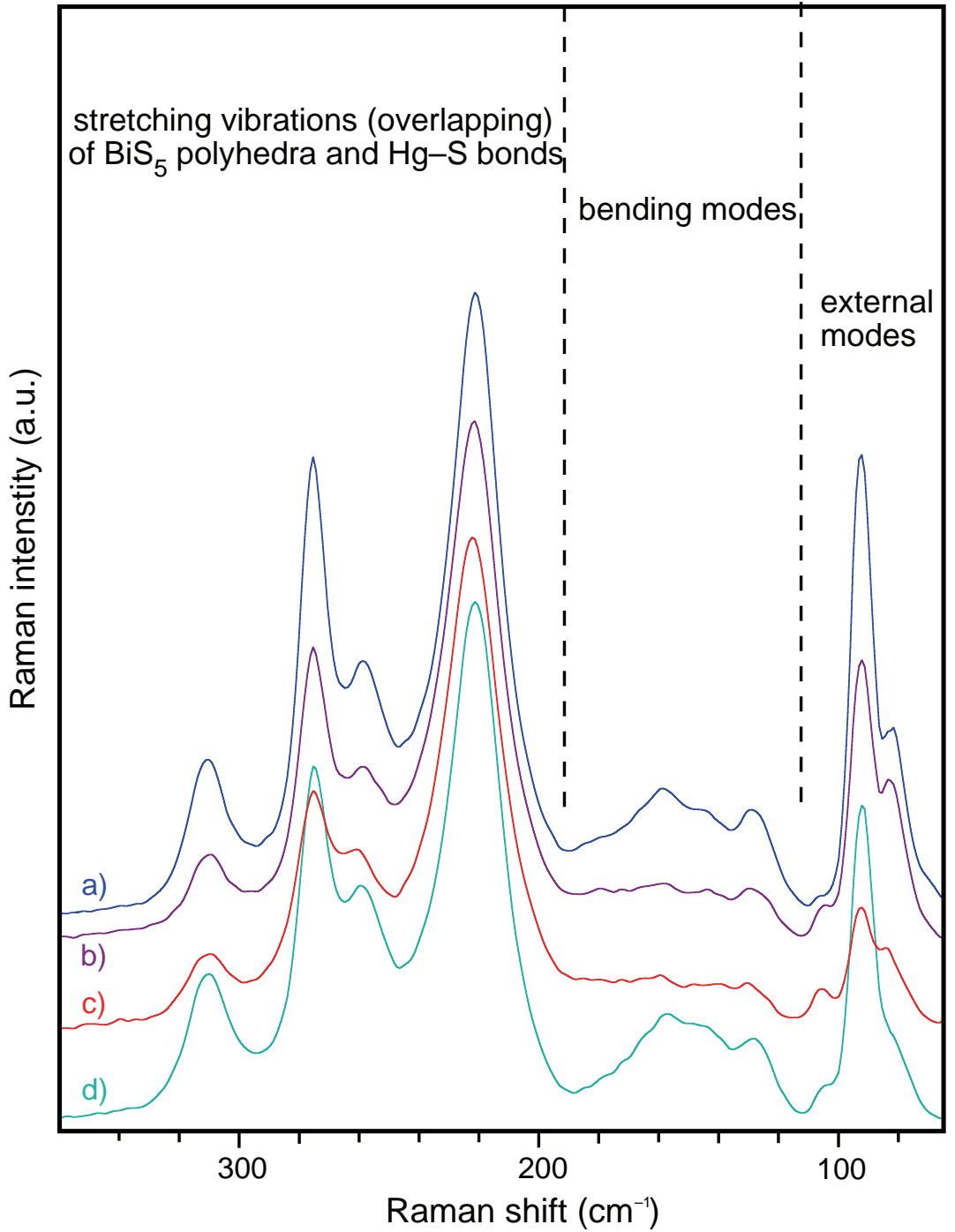

Powder X-ray data acquired from both grumiplucite types (Tab. 3) are very close to each other. Positions of diffraction maxima match the data acquired for this mineral from the Levigliani mine, using $114.6 \mathrm{~mm}$ Gandolfi camera (Orlandi et al. 1998) as well as those for the synthetic $\mathrm{HgBi}_{2} \mathrm{~S}_{4}$ (Brower et al. 1973). No indications of presence of the orthorhombic $\mathrm{MDO}$ polytype (Merlino et al. 2013) have been observed in the studied samples. Comparing the experimental pattern with that calculated from the crystal structure data of grumiplucite (Merlino et al. 2013), significant differences in intensities of individual diffraction maxima were observed. This effect cannot be explained only by a simple preferred sample orientation along [001] (especially for Sb-rich sample). It might be caused by the acicular shape of grumiplucite crystals (Toraya and Marumo 1981). Similar situation was also described for other fibrous sulfosalts - marrucciite (Sejkora et al. 2011), hodrušite (Sejkora et al. 2015) or antimonselite (Škácha et al. 2015).

Fig. 7 Raman spectra of grumiplucite from Rudňany: a) Sb-free; b) 0.13 apfu Sb; c) 0.39 apfu $\mathrm{Sb}$;) $0.70 \mathrm{apfu} \mathrm{Sb}$. 
Tab. 3 X-ray powder diffraction patterns for both types of studied grumiplucite

\begin{tabular}{|c|c|c|c|c|c|c|c|c|c|c|}
\hline & & & \multicolumn{4}{|c|}{ Sb-rich grumiplucite } & \multicolumn{4}{|c|}{ Sb-free grumiplucite } \\
\hline $\mathrm{h}$ & $\mathrm{k}$ & l & $\mathrm{d}_{\text {obs. }}$ & $\mathrm{I}_{\text {obs. }}$ & $\mathrm{I}_{\text {calc. }}$ & $\mathrm{d}_{\text {calc. }}$ & $d_{o b s}$ & $I_{o b s .}$ & $I_{\text {calc. }}$ & $d_{\text {calc. }}$ \\
\hline 2 & 0 & 0 & 6.239 & 1 & 5 & 6.247 & & & & \\
\hline 0 & 0 & 2 & 6.157 & 100 & 26 & 6.158 & 6.153 & 100 & 26 & 6.155 \\
\hline 2 & 0 & $\overline{2}$ & 6.034 & 2 & 2 & 6.042 & 6.044 & 1 & 2 & 6.039 \\
\hline 2 & 0 & 1 & 4.739 & 24 & 23 & 4.740 & 4.739 & 3 & 23 & 4.736 \\
\hline 2 & 0 & $\overline{3}$ & 4.560 & 15 & 14 & 4.561 & 4.557 & 7 & 14 & 4.560 \\
\hline 1 & 1 & 0 & 3.857 & 6 & 12 & 3.856 & & & & \\
\hline 1 & 1 & $\overline{1}$ & 3.845 & 5 & 14 & 3.843 & & & & \\
\hline 2 & 0 & 2 & 3.613 & 82 & 56 & 3.613 & 3.612 & 9 & 56 & 3.610 \\
\hline 4 & 0 & $\overline{2}$ & 3.543 & 59 & 40 & 3.543 & 3.542 & 22 & 40 & 3.540 \\
\hline 1 & 1 & 1 & 3.535 & 23 & 54 & 3.536 & 3.534 & 10 & 54 & 3.534 \\
\hline 2 & 0 & $\overline{4}$ & 3.494 & 93 & 61 & 3.494 & 3.492 & 13 & 61 & 3.493 \\
\hline 4 & 0 & $\overline{1}$ & 3.440 & 5 & 6 & 3.441 & 3.438 & 2 & 6 & 3.437 \\
\hline 4 & 0 & 0 & 3.119 & 7 & 6 & 3.124 & 3.115 & 1 & 6 & 3.121 \\
\hline 0 & 0 & 4 & 3.079 & 69 & 12 & 3.079 & 3.077 & 82 & 12 & 3.077 \\
\hline 3 & 1 & $\overline{2}$ & 3.043 & 55 & 100 & 3.043 & 3.040 & 3 & 100 & 3.042 \\
\hline 4 & 0 & $\overline{4}$ & 3.026 & 30 & 3 & 3.021 & & & & \\
\hline 3 & 1 & 0 & 2.904 & 14 & 46 & 2.905 & 2.905 & 2 & 46 & 2.903 \\
\hline 3 & 1 & $\overline{3}$ & 2.855 & 61 & 52 & 2.857 & 2.856 & 1 & 52 & 2.856 \\
\hline 2 & 0 & $\overline{5}$ & 2.785 & 13 & 4 & 2.785 & 2.783 & 4 & 4 & 2.784 \\
\hline 4 & 0 & 1 & 2.735 & 4 & 4 & 2.735 & & & & \\
\hline 3 & 1 & 1 & 2.639 & 9 & 29 & 2.639 & & & & \\
\hline 4 & 0 & $\overline{5}$ & 2.632 & 4 & 2 & 2.632 & & & & \\
\hline 1 & 1 & $\overline{4}$ & 2.598 & 4 & 12 & 2.598 & 2.596 & 3 & 12 & 2.597 \\
\hline 2 & 0 & 4 & 2.3550 & 47 & 17 & 2.3550 & 2.3538 & 9 & 17 & 2.3534 \\
\hline 6 & 0 & $\overline{2}$ & 2.3361 & 2 & 2 & 2.3366 & & & & \\
\hline 5 & 1 & $\overline{2}$ & 2.3197 & 2 & 10 & 2.3196 & & & & \\
\hline 2 & 0 & $\overline{6}$ & 2.2992 & 39 & 15 & 2.2992 & 2.2979 & 8 & 15 & 2.2984 \\
\hline 1 & 1 & 4 & 2.2517 & 10 & 17 & 2.2511 & 2.2498 & 2 & 17 & 2.2499 \\
\hline 6 & 0 & $\overline{1}$ & 2.2345 & 15 & 16 & 2.2345 & 2.2334 & 2 & 16 & 2.2324 \\
\hline 1 & 1 & $\overline{5}$ & 2.2284 & 8 & 14 & 2.2287 & 2.2281 & 2 & 14 & 2.2278 \\
\hline 6 & 0 & $\overline{5}$ & 2.1771 & 13 & 13 & 2.1774 & 2.1761 & 2 & 13 & 2.1762 \\
\hline 5 & 1 & 0 & 2.1274 & 6 & 21 & 2.1273 & 2.1234 & 1 & 21 & 2.1256 \\
\hline 6 & 0 & 0 & 2.0824 & 5 & 8 & 2.0825 & & & & \\
\hline 5 & 1 & $\overline{5}$ & 2.0753 & 14 & 5 & 2.0758 & 2.0767 & 1 & 5 & 2.0750 \\
\hline 0 & 0 & 6 & 2.0526 & 43 & 9 & 2.0526 & 2.0516 & 59 & 9 & 2.0516 \\
\hline 0 & 2 & 0 & 2.0271 & 16 & 36 & 2.0269 & 2.0269 & 1 & 36 & 2.0262 \\
\hline 6 & 0 & $\overline{6}$ & 2.0137 & 7 & 8 & 2.0139 & 2.0130 & 1 & 8 & 2.0130 \\
\hline 2 & 0 & 5 & 1.9922 & 15 & 4 & 1.9923 & 1.9910 & 3 & 4 & 1.9910 \\
\hline 4 & 0 & $\overline{7}$ & 1.9868 & 7 & 5 & 1.9867 & 1.9864 & 2 & 5 & 1.9861 \\
\hline 1 & 1 & 5 & 1.9511 & 17 & 20 & 1.9506 & 1.9500 & 5 & 20 & 1.9496 \\
\hline 1 & 1 & $\overline{6}$ & 1.9328 & 4 & 8 & 1.9327 & 1.9317 & 2 & 8 & 1.9320 \\
\hline 5 & 1 & $\overline{6}$ & 1.9108 & 15 & 38 & 1.9109 & 1.9104 & 1 & 38 & 1.9102 \\
\hline 2 & 2 & $\overline{3}$ & 1.8541 & 17 & 3 & 1.8523 & & & & \\
\hline 4 & 0 & 4 & 1.8066 & 5 & 3 & 1.8067 & 1.8052 & 1 & 3 & 1.8052 \\
\hline 7 & 1 & $\overline{4}$ & 1.8017 & 8 & 17 & 1.8018 & & & & \\
\hline 5 & 1 & 2 & 1.7992 & 7 & 26 & 1.7994 & 1.7984 & 1 & 26 & 1.7980 \\
\hline 3 & 1 & $\overline{7}$ & 1.7885 & 4 & 11 & 1.7891 & 1.7880 & 1 & 11 & 1.7885 \\
\hline 2 & 2 & 2 & 1.7674 & 10 & 16 & 1.7678 & & & & \\
\hline 4 & 2 & $\overline{2}$ & 1.7603 & 8 & 12 & 1.7594 & & & & \\
\hline 2 & 2 & $\overline{4}$ & 1.7543 & 6 & 18 & 1.7533 & & & & \\
\hline 4 & 0 & $\overline{8}$ & 1.7461 & 8 & 5 & 1.7470 & 1.7462 & 2 & 5 & 1.7465 \\
\hline 8 & 0 & $\overline{5}$ & 1.7446 & 3 & 7 & 1.7440 & & & & \\
\hline 4 & 2 & $\overline{3}$ & 1.7352 & 16 & 3 & 1.7370 & & & & \\
\hline 1 & 1 & $\overline{7}$ & 1.6971 & 6 & 8 & 1.6970 & 1.6961 & 2 & 8 & 1.6963 \\
\hline 0 & 2 & 4 & 1.6922 & 2 & 4 & 1.6930 & 1.6921 & 1 & 4 & 1.6924 \\
\hline 8 & 0 & $\overline{1}$ & 1.6498 & 3 & 5 & 1.6499 & & & & \\
\hline 3 & 1 & 5 & 1.6313 & 1 & 2 & 1.6318 & & & & \\
\hline 4 & 0 & 5 & 1.6005 & 3 & 2 & 1.6007 & 1.5992 & 1 & 2 & 1.5994 \\
\hline 3 & 1 & $\overline{8}$ & 1.5955 & 2 & 6 & 1.5954 & 1.5952 & 1 & 6 & 1.5949 \\
\hline 6 & 0 & 3 & 1.5808 & 7 & 4 & 1.5799 & & & & \\
\hline 8 & 0 & 0 & 1.5620 & 5 & 4 & 1.5619 & & & & \\
\hline 0 & 0 & 8 & 1.5395 & 23 & 5 & 1.5395 & 1.5388 & 32 & 5 & 1.5387 \\
\hline 2 & 2 & $\overline{6}$ & 1.5206 & 5 & 8 & 1.5204 & 1.5199 & 1 & 8 & 1.5199 \\
\hline 6 & 2 & $\overline{5}$ & 1.4832 & 3 & 8 & 1.4836 & & & & \\
\hline 3 & 1 & 6 & 1.4643 & 6 & 8 & 1.4641 & 1.4631 & 3 & 8 & 1.4631 \\
\hline 4 & 0 & 6 & 1.4326 & 12 & 4 & 1.4324 & 1.4318 & 1 & 4 & 1.4313 \\
\hline 8 & 0 & $\overline{9}$ & 1.4129 & 2 & 3 & 1.4125 & 1.4123 & 1 & 3 & 1.4120 \\
\hline 10 & 0 & $\overline{6}$ & 1.4020 & 2 & 1 & 1.4018 & 1.4006 & 1 & 1 & 1.4008 \\
\hline 4 & 0 & $\overline{10}$ & 1.3924 & 7 & 4 & 1.3924 & 1.3918 & 1 & 4 & 1.3919 \\
\hline
\end{tabular}

The refined unit-cell parameters of both types of grumiplucite from Rudňany (Tab. 4) are consistent with those reported previously for grumiplucite or synthetic $\mathrm{HgBi}_{2} \mathrm{~S}_{4}$; the influence of elevated $\mathrm{Sb}$ contents has not been observed.

Raman spectra were collected for grumiplucite crystals with variable Sb contents (Fig. 7). The obtained spectra are, however, very similar; different $\mathrm{Sb}$ occupation does not affect the position and intensity of Raman bands (Tab. 5). They differ significantly from spectra of livingstonite, $\mathrm{HgSb}_{4} \mathrm{~S}_{8}$, a phase with a very similar crystal structure (Fig. 8), though.

In the crystal structure of grumiplucite (Merlino et al. 2013) chains of the edge-sharing squarepyramidal $\left[\mathrm{BiS}_{5}\right]$ polyhedra are paired through the apical $\mathrm{Bi}-\mathrm{S}$ bonds and thus form $\mathrm{Bi}_{2} \mathrm{~S}_{4}$ rods running along the $b$ axis. The rods are interconnected via the short linear $\mathrm{S}-\mathrm{Hg}-\mathrm{S}$ bonds forming structural layers oriented parallel with (001) planes, in agreement with the good $\{001\}$ cleavage of grumiplucite. The crystal structure of grumiplucite shows two $\mathrm{Hg}$, two $\mathrm{Bi}$ and four $\mathrm{S}$ independent positions. The two $\mathrm{Hg}$ atoms have very similar linear coordination by sulphur, with bond distances of $2.36 \AA$ ( $\mathrm{Hg} 1-\mathrm{S} 1$ and $\mathrm{Hg} 2-\mathrm{S} 2$ ); the similar bond-topology occurs in cinnabar with $\mathrm{Hg}-\mathrm{S}$ bond distances of about 2.37 $\AA$ (Auvray and Genet 1973) and in some other Hgsulfosalts (Merlino et al. 2013). The two Bi atoms are characterized by a square-pyramidal $(1+4)$ coordination, with one apical (Bi1-S4 2.62 $\AA$, Bi2-S $2.58 \AA$ ) and four longer basal bonds in the range 2.82-2.86 $\AA$ and 2.84-2.87 $\AA$, respectively (Merlino et al. 2013).

The dominant feature of all grumiplucite spectra measured at different crystals (Fig. 7) is the presence of intense vibration bands in the $320-200 \mathrm{~cm}^{-1}$ region. According to the published papers (Nakamoto 2009; Kharbish et al. 2007, 2009; Škácha et al. 2014), these bands can be assigned to stretching vibrations of the $\left[\mathrm{BiS}_{5}\right]$ polyhedra and $\mathrm{Hg}-\mathrm{S}$ bonds. As the Bi atoms occupy two different crystallographic sites with different internal bond-lengths and, furthermore, the network of linked Bi-polyhedra is not isolated, we were not able to determine and assign the precise character of observed vibration bands. The bands at $275-276$ and $258-261 \mathrm{~cm}^{-1}$ can be connected with vibrations of $\mathrm{Hg}-\mathrm{S}$ bonds, similarly to cinnabar (260-290 and $255 \mathrm{~cm}^{-1}$; Frost et al. 2002). The less intense bands in the region $200-100 \mathrm{~cm}^{-1}$ can be ascribed to bending vibrations of $\mathrm{Hg}-\mathrm{S}$ and $\mathrm{Bi}-\mathrm{S}$ bonds and those below $115 \mathrm{~cm}^{-1}$ should correspond to external modes (translations and librations). 
Tab. 4 Unit-cell parameters of grumiplucite and synthetic $\mathrm{HgBi}_{2} \mathrm{~S}_{4}$ (for monoclinic space group $\mathrm{C} 2 / \mathrm{m}$ )

\begin{tabular}{llllllc}
\hline phase & $a[\AA]$ & $b[\AA]$ & $c[\AA]$ & \multicolumn{1}{c}{$\beta\left[^{\circ}\right]$} & $V\left[\AA^{3}\right]$ & reference \\
\hline grumiplucite $^{\mathrm{a}}$ & $14.183(1)$ & $4.0538(5)$ & $13.980(1)$ & $118.239(1)$ & $708.1(2)$ & this paper \\
grumiplucite $^{\mathrm{b}}$ & $14.172(2)$ & $4.0525(7)$ & $13.975(1)$ & $118.257(8)$ & $707.0(2)$ & this paper \\
grumiplucite $^{14.169(1)}$ & $4.0508(4)$ & $13.975(1)$ & $118.292(6)$ & $706.3(1)$ & Merlino et al. (2013) \\
grumiplucite & $14.164(5)$ & $4.053(1)$ & $13.967(3)$ & $118.28(3)$ & $706.1(3)$ & Orlandi et al. (1998) \\
synthetic & $14.17(1)$ & $4.06(1)$ & $13.99(1)$ & $118.27(1)$ & 708.9 & Mumme and Watts (1980) \\
\hline
\end{tabular}

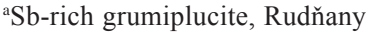

${ }^{\mathrm{b}} \mathrm{Sb}$-free grumiplucite, Rudňany

Tab. 5 Band components of the Raman spectra of grumiplucite with various Sb contents

\begin{tabular}{|c|c|c|c|c|c|c|c|c|c|c|c|}
\hline \multicolumn{3}{|c|}{ Sb-free } & \multicolumn{3}{|c|}{0.13 apfu Sb } & \multicolumn{3}{|c|}{0.39 apfu Sb } & \multicolumn{3}{|c|}{0.70 apfu $\mathrm{Sb}$} \\
\hline $\mathrm{BP}$ & $\mathrm{I}_{\text {rel. }}$ & FWHM & BP & $\mathrm{I}_{\text {rel. }}$ & FWHM & BP & $\mathrm{I}_{\text {rel. }}$ & FWHM & $\mathrm{BP}$ & $\mathrm{I}_{\text {rel. }}$ & FWHM \\
\hline 310 & 32 & 9 & 310 & 14 & 9 & 310 & 12 & 9 & 310 & 26 & 10 \\
\hline 275 & 65 & 4 & 276 & 47 & 3 & 275 & 35 & 2 & 275 & 54 & 2 \\
\hline 258 & 29 & 12 & 259 & 21 & 13 & 261 & 23 & 12 & 259 & 35 & 11 \\
\hline 221 & 100 & 6 & 222 & 100 & 6 & 222 & 100 & 7 & 221 & 100 & 11 \\
\hline 162 & 16 & 15 & 163 & 9 & 15 & 163 & 8 & 15 & 160 & 20 & 18 \\
\hline 144 & 9 & 10 & 144 & 6 & 9 & 143 & 8 & 9 & 143 & 11 & 11 \\
\hline \multirow[t]{2}{*}{127} & 13 & 9 & 128 & 9 & 9 & 129 & 7 & 9 & 127 & 16 & 11 \\
\hline & & & 104 & 5 & 4 & 105 & 6 & 5 & 105 & 6 & 5 \\
\hline 92 & 69 & 8 & 92 & 50 & 7 & 93 & 20 & 7 & 92 & 57 & 6 \\
\hline 82 & 29 & 7 & 82 & 29 & 8 & 83 & 15 & 9 & 82 & 19 & 13 \\
\hline
\end{tabular}

FWHM $=$ Full-width and half-maximum

$\mathrm{BP}$ (band position in $\left.\mathrm{cm}^{-1}\right), \mathrm{I}_{\text {rel. }}$ (relative intensity in \%), FWHM $\left(\mathrm{cm}^{-1}\right)$

Tab. 6 Chemical composition of members of bismuthinite-stibnite solid solution (in wt. \% and apfu)

\begin{tabular}{|c|c|c|c|c|c|c|c|c|c|c|c|c|}
\hline & \multicolumn{8}{|c|}{ Sb-rich bismuthinite } & \multicolumn{4}{|c|}{ Bi-rich stibnite } \\
\hline & mean & 1 & 2 & 3 & 4 & 5 & 6 & 7 & 1 & 2 & 3 & 4 \\
\hline Ig & 1.63 & 1.74 & 1.75 & 1.7 & 1.82 & 8 & 1.89 & 0.52 & 0.52 & כני & S & 0.90 \\
\hline $\mathrm{Cu}$ & & 0.06 & & 0.06 & 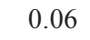 & & & & .00 & 0.07 & 0.07 & 0.08 \\
\hline b & 1 & 17.79 & 2 & 17.69 & 1 & & & & & 47.82 & & 54.91 \\
\hline $3 i$ & & 58.91 & 59.66 & 59.06 & 58 & 58 & & & 93 & 26.45 & 33.70 & 18.17 \\
\hline Is & & & & & & & & & & & & 0.15 \\
\hline & 21.45 & 21.46 & 21.48 & 21.38 & 21.44 & 21.51 & 21.56 & 21 & 24.66 & 25.23 & 24.29 & 26.03 \\
\hline total & 100.08 & 100.24 & 100.71 & 100.27 & 99.78 & 100.49 & 99.99 & 99.08 & 99.80 & 100.17 & 99.50 & 100.24 \\
\hline $\mathrm{Hg}$ & .037 & 0.039 & 0.039 & 0.040 & 0.041 & & & & & 0.010 & 0.012 & 0.017 \\
\hline $\mathrm{Cu}$ & 003 & .004 & .000 & .004 & 0.004 & 0.008 & 0.000 & 0 . & 00 & 0.004 & 0.004 & 0.004 \\
\hline $\mathrm{Sb}$ & 0.657 & 0.658 & $\begin{array}{ll}3 & 0.647\end{array}$ & 0.655 & 0.661 & & & & & $5 \quad 1.498$ & 1.332 & \\
\hline $\boldsymbol{H}$ & & 1.269 & 1.284 & 1.275 & 1.260 & 57 & & & 38 & 0.483 & 0.641 & 0.320 \\
\hline As & 0.015 & 0.017 & $7 \quad 0.018$ & 0.018 & 0.016 & & 0.020 & & 0.007 & $\begin{array}{l}7 \\
0.004 \\
\end{array}$ & 0.000 & 0.00 \\
\hline$M$ & 1.984 & 1.987 & $7 \quad 1.988$ & 1.992 & 1.982 & & & & 2.010 & 1.999 & 1.990 & 2.010 \\
\hline & 3.016 & 3.013 & 33.012 & 3.008 & 3.018 & 3.012 & 3.022 & 3.029 & 2.990 & 3.001 & 3.010 & 2.99 \\
\hline
\end{tabular}

Empirical formulae were calculated on the basis of 5 apfu

\subsection{Minerals of bismuthinite-stibnite solid solution}

Minerals of the bismuthinite-stibnite solid solution were found in the studied association only rarely as microscopic aggregates and individual crystals (up to $100 \mu \mathrm{m}$ in size) usually overgrown by younger cin- nabar. Aggregates of Sb-rich bismuthinite are homogenous with $\mathrm{Sb}$ content in the range of 0.65-0.66 apfu (Fig. 9) and unusually small contents of $\mathrm{Hg}$ (up to $0.04 \mathrm{apfu}$ ) and As (up to 0.02 apfu). Its chemical composition (Tab. 6) can be written as $\left(\mathrm{Bi}_{1.27} \mathrm{Sb}_{0.66} \mathrm{Hg}_{0.04} \mathrm{As}_{0.02}\right)_{\Sigma 1.99} \mathrm{~S}_{3.02}$ on the basis of 5 apfu. The Bi-rich stibnite forms strongly zoned aggregates (Fig. 5) with variable $\mathrm{Bi}$ contents $(0.32-0.64$ apfu; Fig. 9) and minor contents of $\mathrm{Hg}$ (up to $0.02 \mathrm{apfu}$ ) and $\mathrm{As}$ (up to $0.01 \mathrm{apfu}$ ). The coefficients of empirical formulae of Bi-rich stibnite are given in Tab. 6.

\subsection{Cinnabar}

Cinnabar is the most abundant sulfide mineral in the studied mineral association. It forms irregular aggregates up to $5 \mathrm{~cm}$ in size as well as perfectly developed and often twinned striated rhombohedral crystals up to $3 \mathrm{~mm}$ or, unusual acicular, tabular or prismatic crystals up to $2 \mathrm{~mm}$ in size. Identity of all morphological forms of cinnabar was confirmed by PXRD. Color of cinnabar crystals and aggregates is dark red to black with reddish internal reflection. Chemical composition of all morphological forms is well comparable; only minor contents of Ag (up to $0.006 \mathrm{apfu}$ ), $\mathrm{Fe}$ (up to $0.018 \mathrm{apfu}$ ), $\mathrm{Cu}$ (up to $0.003 \mathrm{apfu}$ ) and $\mathrm{Bi}$ (up to 0.005 apfu) were determined (Tab. 7).

\subsection{Hg-rich tetrahedrite}

Hg-rich tetrahedrite is the youngest mineral phase in studied mineral association. It forms steel grey crystalline aggregates and small crystals up to $1 \mathrm{~mm}$ in size growing on earlier grumiplucite (Fig. 4d) or cinnabar crystals. A general formula of the tetrahedrite group minerals can be expressed according to Moëlo et al. (2008), as ${ }^{[\mathrm{III}]} A_{6}{ }^{[\mathrm{IV}]}(B, C)_{6}^{[\mathrm{III}]} X_{4}^{[\mathrm{IV}]} Y_{12}{ }^{[\mathrm{VI}]}$ 
Fig. 8 Raman spectrum of livingstonite from Huitzuco (Mexico) with empirical formula $\mathrm{Hg}_{1.01}\left(\mathrm{Sb}_{3.89} \mathrm{As}_{0.08}\right)_{\Sigma 3.97} \mathrm{~S}_{8.01}$.

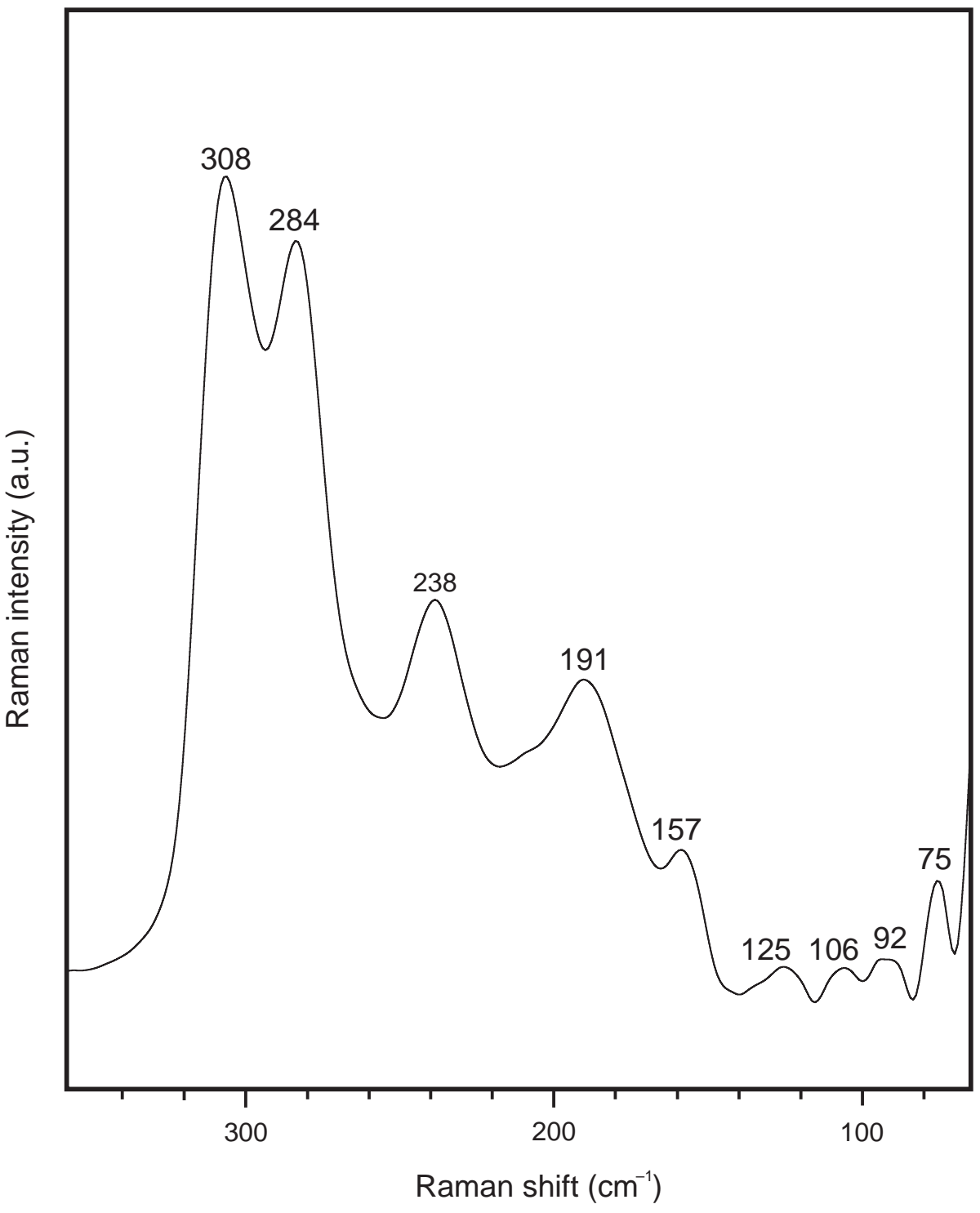

Tab. 7 Chemical composition of cinnabar (in wt. \% and apfu)

\begin{tabular}{|c|c|c|c|c|c|c|c|c|c|c|c|c|}
\hline & mean & range & 1 & 2 & 3 & 4 & 5 & 6 & 7 & 8 & 9 & 10 \\
\hline $\mathrm{Ag}$ & 0.13 & $0.00-0.29$ & 0.21 & 0.18 & 0.16 & 0.16 & 0.13 & 0.13 & 0.00 & 0.10 & 0.22 & 0.15 \\
\hline $\mathrm{Fe}$ & 0.03 & $0.00-0.43$ & 0.00 & 0.00 & 0.00 & 0.00 & 0.00 & 0.00 & 0.00 & 0.00 & 0.22 & 0.16 \\
\hline $\mathrm{Hg}$ & 84.87 & $84.03-86.45$ & 85.14 & 85.09 & 86.45 & 85.40 & 85.23 & 85.13 & 85.19 & 84.95 & 84.93 & 85.01 \\
\hline $\mathrm{Cu}$ & 0.01 & $0.00-0.10$ & 0.00 & 0.00 & 0.00 & 0.06 & 0.00 & 0.00 & 0.00 & 0.00 & 0.06 & 0.08 \\
\hline $\mathrm{Bi}$ & 0.10 & $0.00-0.47$ & 0.41 & 0.21 & 0.00 & 0.18 & 0.00 & 0.00 & 0.00 & 0.26 & 0.00 & 0.00 \\
\hline $\mathrm{S}$ & 13.97 & $13.66-14.14$ & 14.08 & 14.02 & 13.98 & 14.05 & 14.00 & 14.06 & 14.03 & 14.04 & 13.72 & 13.95 \\
\hline total & 99.11 & & 99.84 & 99.50 & 100.59 & 99.84 & 99.36 & 99.31 & 99.22 & 99.34 & 99.16 & 99.34 \\
\hline$\overline{\mathrm{Ag}}$ & 0.0028 & & 0.0045 & 0.0039 & 0.0035 & 0.0033 & 0.0028 & 0.0027 & 0.0000 & 0.0020 & 0.0048 & 0.0032 \\
\hline $\mathrm{Fe}$ & 0.0014 & & 0.0000 & 0.0000 & 0.0000 & 0.0000 & 0.0000 & 0.0000 & 0.0000 & 0.0000 & 0.0092 & 0.0064 \\
\hline $\mathrm{Hg}$ & 0.9824 & & 0.9787 & 0.9817 & 0.9924 & 0.9819 & 0.9850 & 0.9824 & 0.9851 & 0.9808 & 0.9867 & 0.9808 \\
\hline $\mathrm{Cu}$ & 0.0004 & & 0.0000 & 0.0000 & 0.0000 & 0.0020 & 0.0000 & 0.0000 & 0.0000 & 0.0000 & 0.0023 & 0.0029 \\
\hline $\mathrm{Bi}$ & 0.0011 & & 0.0045 & 0.0023 & 0.0000 & 0.0020 & 0.0000 & 0.0000 & 0.0000 & 0.0028 & 0.0000 & 0.0000 \\
\hline$\overline{\Sigma M e}$ & 0.9881 & & 0.9877 & 0.9880 & 0.9958 & 0.9892 & 0.9879 & 0.9852 & 0.9851 & 0.9857 & 1.0030 & 0.9933 \\
\hline$S$ & 1.0118 & & 1.0123 & 1.0120 & 1.0042 & 1.0108 & 1.0121 & 1.0148 & 1.0149 & 1.0143 & 0.9970 & 1.0067 \\
\hline
\end{tabular}

Mean and range for all 38 spot analyses, 1-10 representative analyses of cinnabar from Rudňany.

Empirical formulae were calculated on the basis of $2 \mathrm{apfu}$ 


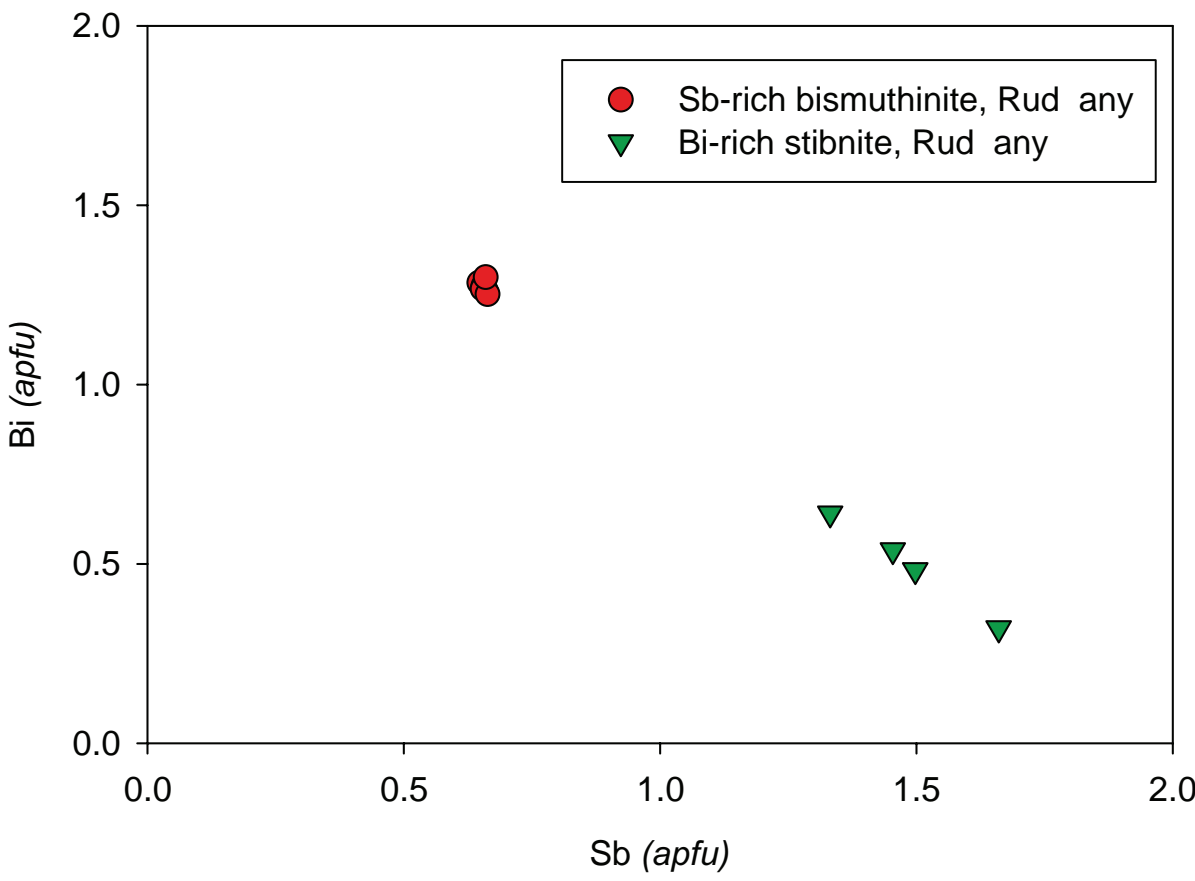

Fig. $9 \mathrm{Sb}$ vs. Bi (apfu) graph for members of bismuthinite-stibnite solid solution from Rudňany.
$Z_{1}$, where $A=\mathrm{Cu}, \mathrm{Ag} ; B=\mathrm{Cu}, \mathrm{Ag}$ and $C$ is generally divalent metal (typically $\mathrm{Fe}, \mathrm{Zn}$, but also $\mathrm{Hg}, \mathrm{Mn}, \mathrm{Cd}$, $\mathrm{Cu}$ etc.) in the same coordination as $B ; X=\mathrm{Sb}, \mathrm{As}, \mathrm{Bi}$, Te; $Y$ and $Z=\mathrm{S}$ and Se. In the analysed samples, copper predominates at the $A$ site and only minor contents of $\mathrm{Ag}$ (up to $0.05 \mathrm{apfu}$ ) were detected; then the $B$ position is fully occupied by $\mathrm{Cu}$. Dominant representation of $\mathrm{Hg}$
(1.31-2.08 apfu) is characteristic of the divalent $C$ position along with minor contents of Fe and $\mathrm{Zn}$ (Fig. 10). Antimony occupies the majority of $X$ position; minor contents of As (up to $0.89 \mathrm{apfu}$ ) and $\mathrm{Bi}$ (up to $0.11 \mathrm{apfu}$ ) were also detected (Fig. 11). Overall occupation of $\mathrm{Sb}+$ As + Bi position in the range 4.17-4.37 apfu is unusual. The $Y$ and $Z$ positions are fully occupied by sulfur. Av-

Tab. 8 Chemical composition of Hg-rich tetrahedrite (in wt. \% and apfu)

\begin{tabular}{|c|c|c|c|c|c|c|c|c|c|c|c|c|}
\hline & mean & range & 1 & 2 & 3 & 4 & 5 & 6 & 7 & 8 & 9 & 10 \\
\hline$\overline{\mathrm{Ag}}$ & 0.17 & $0.00-0.26$ & 0.22 & 0.22 & 0.20 & 0.23 & 0.16 & 0.20 & 0.20 & 0.00 & 0.10 & 0.00 \\
\hline $\mathrm{Fe}$ & 0.74 & $0.10-1.05$ & 0.68 & 0.73 & 0.91 & 0.89 & 0.93 & 1.02 & 0.66 & 0.49 & 0.11 & 0.10 \\
\hline $\mathrm{Zn}$ & 0.54 & $0.00-1.05$ & 0.22 & 0.29 & 0.41 & 0.98 & 1.00 & 1.01 & 0.51 & 0.21 & 0.00 & 0.12 \\
\hline $\mathrm{Hg}$ & 16.75 & $14.48-20.93$ & 17.84 & 17.19 & 16.39 & 15.36 & 14.51 & 14.53 & 16.58 & 18.78 & 20.71 & 20.93 \\
\hline $\mathrm{Cu}$ & 32.98 & $30.09-34.24$ & 32.83 & 33.02 & 33.74 & 33.99 & 34.03 & 34.24 & 32.99 & 30.99 & 31.89 & 31.58 \\
\hline $\mathrm{Sb}$ & 24.46 & $21.65-25.99$ & 24.70 & 24.87 & 22.91 & 22.10 & 23.72 & 22.90 & 24.99 & 23.54 & 25.73 & 25.67 \\
\hline $\mathrm{Bi}$ & 0.73 & $0.00-1.19$ & 1.19 & 1.04 & 0.91 & 0.72 & 0.66 & 0.70 & 1.02 & 0.20 & 0.00 & 0.00 \\
\hline As & 1.89 & $0.00-3.69$ & 1.45 & 1.42 & 2.97 & 3.69 & 2.77 & 3.34 & 1.66 & 1.89 & 0.09 & 0.00 \\
\hline $\mathrm{S}$ & 22.43 & $20.71-23.12$ & 21.93 & 21.92 & 22.65 & 22.99 & 23.07 & 23.12 & 22.13 & 22.63 & 20.77 & 20.71 \\
\hline total & 100.69 & & 101.06 & 100.68 & 101.09 & 100.95 & 100.83 & 101.06 & 100.73 & 98.74 & 99.39 & 99.11 \\
\hline $\mathrm{Ag}$ & 0.030 & & 0.038 & 0.038 & 0.034 & 0.038 & 0.027 & 0.033 & 0.035 & 0.000 & 0.017 & 0.000 \\
\hline $\mathrm{Cu}$ & 9.682 & & 9.761 & 9.807 & 9.773 & 9.722 & 9.733 & 9.743 & 9.739 & 9.312 & 9.911 & 9.859 \\
\hline $\mathrm{A}+\mathrm{B}$ & 9.711 & & 9.799 & 9.844 & 9.807 & 9.760 & 9.760 & 9.777 & 9.773 & 9.312 & 9.929 & 9.859 \\
\hline $\mathrm{Fe}$ & 0.247 & & 0.229 & 0.246 & 0.299 & 0.288 & 0.303 & 0.331 & 0.221 & 0.168 & 0.038 & 0.037 \\
\hline $\mathrm{Zn}$ & 0.155 & & 0.064 & 0.084 & 0.116 & 0.272 & 0.277 & 0.280 & 0.147 & 0.062 & 0.000 & 0.036 \\
\hline $\mathrm{Hg}$ & 1.557 & & 1.680 & 1.617 & 1.503 & 1.392 & 1.314 & 1.310 & 1.551 & 1.788 & 2.039 & 2.070 \\
\hline $\mathrm{C}$ & 1.960 & & 1.973 & 1.948 & 1.918 & 1.952 & 1.895 & 1.921 & 1.919 & 2.018 & 2.077 & 2.143 \\
\hline$\overline{\mathrm{Sb}}$ & 3.747 & & 3.833 & 3.856 & 3.463 & 3.299 & 3.541 & 3.400 & 3.851 & 3.692 & 4.174 & 4.184 \\
\hline $\mathrm{Bi}$ & 0.065 & & 0.108 & 0.094 & 0.080 & 0.062 & 0.057 & 0.061 & 0.091 & 0.018 & 0.000 & 0.000 \\
\hline As & 0.471 & & 0.365 & 0.356 & 0.730 & 0.895 & 0.671 & 0.806 & 0.416 & 0.482 & 0.023 & 0.000 \\
\hline $\mathrm{X}$ & 4.283 & & 4.306 & 4.306 & 4.273 & 4.256 & 4.270 & 4.267 & 4.358 & 4.192 & 4.197 & 4.184 \\
\hline$\underline{S}$ & 13.046 & & 12.922 & 12.902 & 13.002 & 13.032 & 13.075 & 13.036 & 12.949 & 13.479 & 12.797 & 12.814 \\
\hline
\end{tabular}

Mean and range for all 25 spot analyses, 1-10 representative analyses of Hg-rich tetrahedrite from Rudňany.

Empirical formulae were calculated on the basis of $29 a p f u$. 
Fig. $10 \mathrm{Hg}$ vs. $\mathrm{Fe}+\mathrm{Zn}(a p f u)$ graph for Hg-rich tetrahedrite from Rudňany.

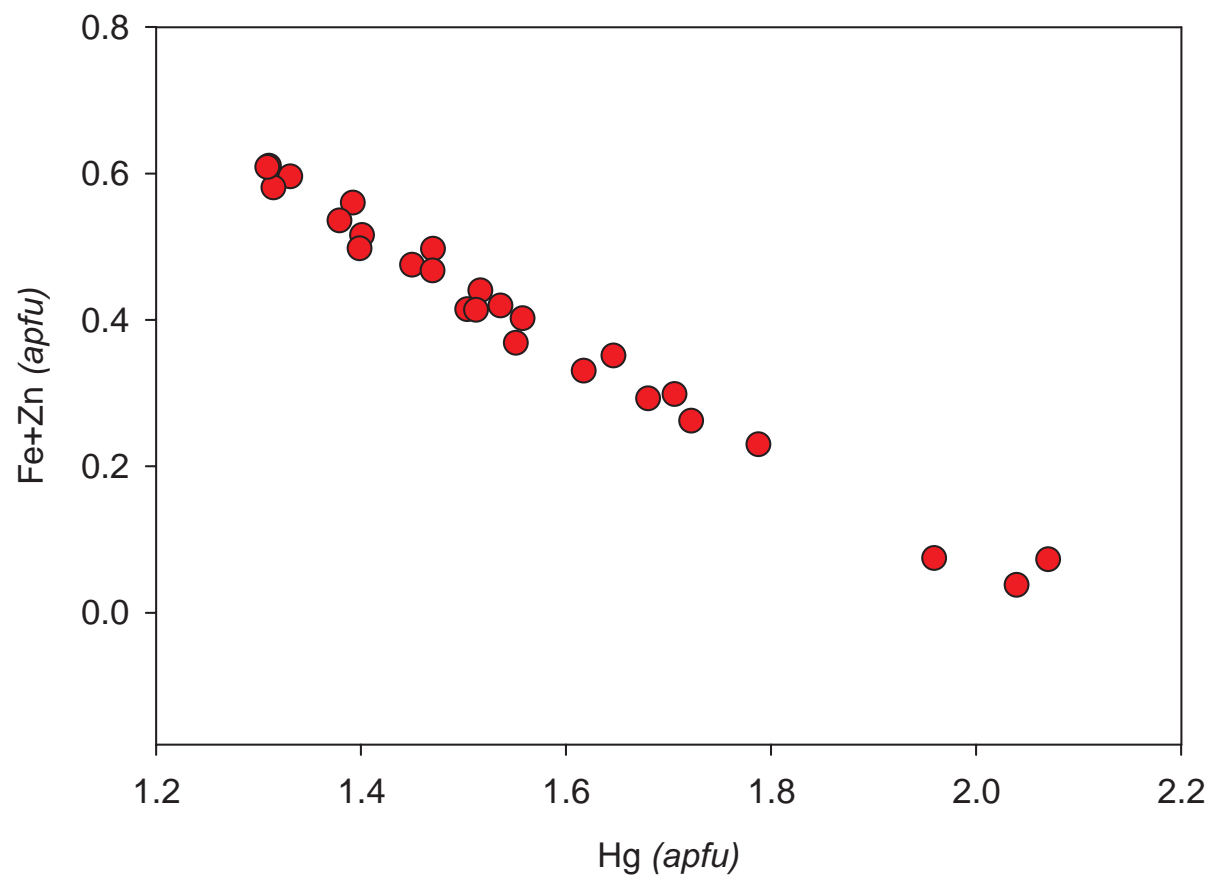

erage chemical composition of the Hg-rich tetrahedrite from the studied association (Tab. 8) is $\left(\mathrm{Cu}_{5.97} \mathrm{Ag}_{0.03}\right)_{\Sigma 6.00}$ $\mathrm{Cu}_{3.71}\left(\mathrm{Hg}_{1.56} \mathrm{Fe}_{0.24} \mathrm{Zn}_{0.16}\right)_{\Sigma 1.96}\left(\mathrm{Sb}_{3.75} \mathrm{As}_{0.47} \mathrm{Bi}_{0.07}\right)_{\Sigma 4.29} \mathrm{~S}_{13.05}$ on the basis of 29 apfu. Chemistry of the studied Hg-rich tetrahedrite is close to the data for Hg-rich tetrahedrites from the Rudňany deposit (samples D14, D15, D16) recently published by Velebil (2014).

\section{Conclusions}

A second world-occurrence of the very rare $\mathrm{Hg}$-sulfosalt, grumiplucite, is reported from the Rudňany siderite-type ( $\mathrm{Fe}-$ $\mathrm{Ba}-\mathrm{Cu}-\mathrm{Hg}$ ) deposit, Slovakia. New analytical data from PXRD, EMPA-WDS and Raman spectroscopy are given for grumiplucite, which is one of the youngest ore minerals at the studied site. It occurs in drusy cavities of siderite and it is closely associated with minerals of bismuthinite-stibnite solid solution and younger crystals of cinnabar and $\mathrm{Hg}$ rich tetrahedrite.

Fig. $11 \mathrm{Sb}$ vs. As, Bi (apfu) graph for $\mathrm{Hg}$-rich tetrahedrite from Rudňany.
Acknowledgements. This work was financially supported by the SYNTHESYS project (CZ-TAF-3450) to MŠ and by the Ministry of Culture of the Czech Republic (DKRVO 2015/02; National Museum 00023272) to JS. Both the referees, Jakub Plášil and František Veselovský, as well as handling editor František Laufek and editorin-chief Vojtěch Janoušek, are highly acknowledged for comments and suggestions that helped greatly to improve the manuscript.

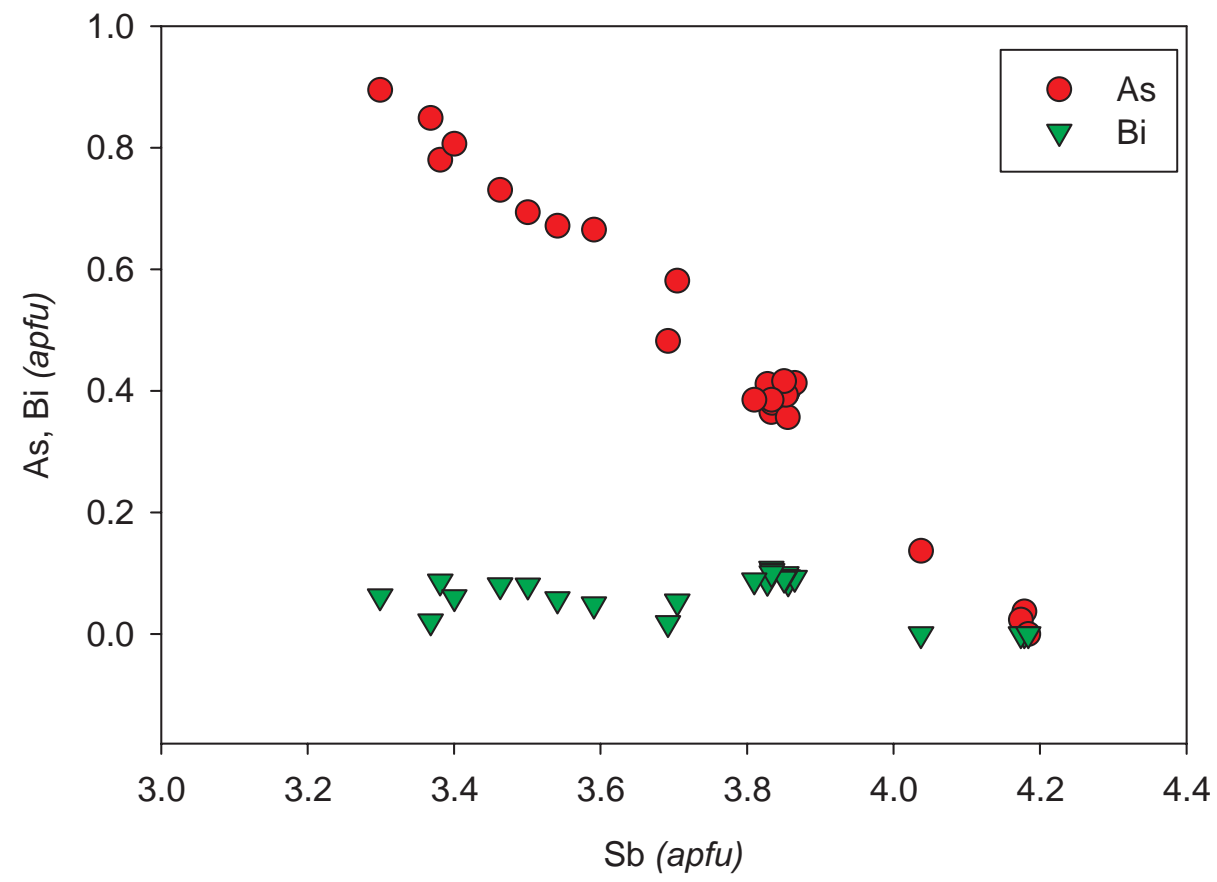




\section{References}

Auvray P, Genet F (1973) Affinement de la structure cristalline du cinabre $\alpha-\mathrm{HgS}$. Bull Soc fr Minéral Cristallogr 96: 218-219 (in French)

BERNARD JH (1955) Contribution to the sequence of minerals of the Droždiak vein in Rudňany. Věst Ústř Úst geol 30: 237-239 (in Czech)

Bernard JH (1961) Mineralogie und Geochemie der Siderit-Schwerspatgänge mit Sulfiden im Gebiet von Rudňany (Tschechoslowakei). Geol Práce 58: 1-222

Brower WS, Parker HS, Roth RS (1973) Synthesis of mercury bismuth sulfide. Mat Res Bull 8: 859-862

Burnham CW (1962) Lattice constant refinement. Carnegie Institute Washington Yearbook 61: 132-135

Cambel B, Jarkovský J, Faith L, Forgáč J, Hovorka D, Hrnčárová M, Hurný J, Ivan P, Karoli A, Král J, Litavec J, Matula I, Mihalov J, Popreñák J, Rényi K, Rojkovič I, Rozložník L, SASvári T, SAVČenková L, Spišiak J, ŠMejKal V, Turan J, Turanová L, VArČEK C, ŽABKA M, ŽUKOV F (1985) The Rudňany ore field-geochemical-metallogenic characteristics. Veda, Bratislava, pp 1-363 (in Slovak)

ĎuñA R, OzDín D (2012) Minerals of Slovakia. Granit, Praha, pp 1-480 (in Slovak)

Frost RL, Martens WN, Kloprogge JT (2002) Raman spectroscopic study of cinnabar $(\mathrm{HgS})$, realgar $\left(\mathrm{As}_{4} \mathrm{~S}_{4}\right)$, and orpiment $\left(\mathrm{As}_{2} \mathrm{~S}_{3}\right)$ at 298 and $77 \mathrm{~K}$. Neu Jb Mineral, Mh 10: 469-480

Grecula P, Abonyi A, Abonyiová M, Antaš J, Bartalský B, Bartalský J, DianišKa I, Ďuña R, Gargulák M, GAZDAČKo L', HudÁČEK J, KobUlskÝ J, LÖRINCZ L, Macko J, NÁvesñák D, Németh Z, Novotný L, Radvanec M, RoJKovič I, RozložNík L, VARČEK C, Zlocha Z (1995) Mineral Deposits of the Slovak Ore Mountains. Volume 1. Geocomplex, Bratislava, pp 1-834

Hurai V, GazdačKo L', Ferenčíková E, Majzlan J, RepČoK I (1998) Origin of ore-forming fluids of the vein siderite deposits Gretla, Jedl'ovec and Rudňany (Spišsko-gemerské Rudohorie Mts.). Miner Slov 30: 423-430 (in Slovak)

Hurai V, Harčová E, Huraiová M, Ozdín D, Prochaska W, Wiegerová V (2002) Origin of siderite veins in the Western Carpathians I. $P-T-X-\delta^{13} \mathrm{C}-\delta^{18} \mathrm{O}$ relations in ore-forming brines of the Rudňany deposits. Ore Geol Rev 21: 67-101

HurnÝ J, KrišTín J (1978) Some Ni-Co minerals from the Rudňany deposit (Zlatník vein - Spišsko-gemerské Rudohorie Mts.). Miner Slov 10: 221-238

Kharbish S, Libowitzky E, Beran A (2007) The effect of As-Sb substitution in the Raman spectra of tetrahedritetennantite and pyrargyrite-proustite solid solution. Eur J Mineral 19: 567-574

Kharbish S, Libowitzky E, Beran A (2009) Raman spectra of isolated and interconnected pyramidal $\mathrm{XS}_{3}$ groups
$(\mathrm{X}=\mathrm{Sb}, \mathrm{Bi})$ in stibnite, bismuthinite, kermesite, stephanite and bournonite. Eur J Mineral 21: 325-333

Merlino S, Biagioni C, Orlandi P (2013) The crystal structure of grumiplucite: its OD character and structural relationships. Rend Fis Acc Lincei 24: 47-52

Moëlo Y, Makovicky E, Mozgova NN, Jambor JL, Cook N, Pring A, Paar W, Nickel EH, Graeser S, Karup-Møller S, Balić-Žunić T, Mumme WF, Vurro F, Topa D, Bindi L, Bente K, Smizu M (2008) Sulfosalt systematics: a review report of the sulfosalt sub-committee of the IMA commission on ore mineralogy. Eur J Mineral 20: 7-46

Mumme WG, Watts JA (1980) $\mathrm{HgBi}_{2} \mathrm{~S}_{4}$ : crystal structure and relationship with the pavonite homologous series. Acta Cryst B36: 1300-1304

NAкамото K (2009) Infrared and Raman Spectra of Inorganic and Coordination Compounds. $6^{\text {th }}$ Ed. John Wiley and Sons, New Jersey, pp 1-419

ONDRUŠ P (1993) ZDS - a computer program for analysis of X-ray powder diffraction patterns. Materials Science Forum, EPDIC-2, Enchede, 133-136: 297-300

Orlandi P, Dini A, Olmi F (1998) Grumiplucite, a new mercury-bismuth sulfosalt species from the Levigliani mine, Apuan Alps, Tuscany, Italy. Canad Mineral 36: 1321-1326

Pouchou JL, Pichoir F (1985) "PAP” ( $\varphi \rho Z)$ procedure for improved quantitative microanalysis. In: ArMSTRONG JT (ed) Microbeam Analysis. San Francisco Press 104-106

RADVANEC M, Grecula P, ŽÁK K (2004) Siderite mineralization of the Gemericum Superunit (Western Carpathians, Slovakia): review and a revised genetic model. Ore Geol Rev 24: 267-298

RoJKovič I (1977) Mineralogical and geochemical study on opaque minerals of the Rudňany deposit. Unpublished manuscript, ŠGÚDŠ-Geofond, Bratislava, pp 1-232 (in Slovak)

Sejkora J, Ozdín D, Laufek F, PlášIl J, Litochleb J (2011) Marrucciite, a rare $\mathrm{Hg}$-sulfosalt from the Gelnica ore deposit (Slovak Republic), and its comparison with the type occurrence at Buca della Vena mine (Italy). J Geosci 56: $399-408$

SeJkora J, Števko M, Ozdín D, PrŠek J, Jeleň S (2015) Unusual morphological forms of hodrušite from the Rozália vein, Hodruša-Hámre near Banská Štiavnica (Slovak Republic). J Geosci 60: 11-22

Š́nácha P, Buixaderas E, Plášil J, Sejkora J, Goliáš V, VLČEK V (2014) Permingeatite, $\mathrm{Cu}_{3} \mathrm{SbSe}_{4}$, from Př́bram (Czech Republic): description and Raman spectroscopy investigations of the luzonite-subgroup of minerals. Canad Mineral 52: 501-511

ŠKÁcha P, PláŠIl J, SEJKora J, Goliáš V (2015) Sulfur-rich antimonselite, $\mathrm{Sb}_{2}(\mathrm{Se}, \mathrm{S})_{3}$, in the Se-bearing mineral association from the Príbram uranium and base metal ore district, Czech Republic. J Geosci 60: 23-29

Toraya H, Marumo F (1981) Preferred orientation correction in powder pattern fitting. Mineral J 10: 211-221 
Velebil D (2014) A contribution to knowledge of chemistry of mercurian tetrahedrites: localities Jedová hora (Czech Republic), Rudňany, Rožňava, Nižná Slaná, Slovinky (Slovakia) and Maškara (Bosna and Herzegovina). Bull mineral-petrolog Odd Nár Muz (Praha) 22: $131-143$
VozÁrová A, VozÁr J (1988) Late Paleozoic in West Carpathians. GÚDŠ, Bratislava, pp 1-314

ŽÁk K, Radvanec M, Grecula P, Bartalský B (1991) Sr, S, $\mathrm{C}, \mathrm{O}$ isotopes and metamorphic-hydrothermal model of vein mineralization, Gemeric Unit, Western Carpathians. Miner Slov 23: 95-108 (in Slovak) 